ARTICLE

\title{
Oxygen-sensitive methylation of ULK1 is required for hypoxia-induced autophagy
}

Jingyi Li,2,10, Tao Zhang (10 1,2,10, Tao Ren ${ }^{3,10}$, Xiaoyu Liao ${ }^{4}$, Yilong Hao ${ }^{5}$, Je Sun Lim ${ }^{6}$, Jong-Ho Lee ${ }^{6,7}, \mathrm{Mi} \mathrm{Li}^{8,9}$, Jichun Shao ${ }^{1} \&$ Rui Liu (iD ${ }^{4 凶}$

Hypoxia is a physiological stress that frequently occurs in solid tissues. Autophagy, a ubiquitous degradation/recycling system in eukaryotic cells, renders cells tolerant to multiple stressors. However, the mechanisms underlying autophagy initiation upon hypoxia remains unclear. Here we show that protein arginine methyltransferase 5 (PRMT5) catalyzes symmetrical dimethylation of the autophagy initiation protein ULK1 at arginine 170 (R170me2s), a modification removed by lysine demethylase 5C (KDM5C). Despite unchanged PRMT5mediated methylation, low oxygen levels decrease KDM5C activity and cause accumulation of ULK1 R170me2s. Dimethylation of ULK1 promotes autophosphorylation at T180, a prerequisite for ULK1 activation, subsequently causing phosphorylation of Atg13 and Beclin 1, autophagosome formation, mitochondrial clearance and reduced oxygen consumption. Further, expression of a ULK1 R170K mutant impaired cell proliferation under hypoxia. This study identifies an oxygen-sensitive methylation of ULK1 with an important role in hypoxic stress adaptation by promoting autophagy induction.

\footnotetext{
${ }^{1}$ The Second Affiliated Hospital of Chengdu Medical College, China National Nuclear Corporation 416 Hospital, Chengdu 610051, China. ${ }^{2}$ School of Biological Sciences and Technology, Chengdu Medical College, Chengdu 610599, China. ${ }^{3}$ Oncology Department, Clinical Medical College and The First Affiliated Hospital of Chengdu Medical College, Chengdu 610500, China. ${ }^{4}$ State Key Laboratory of Oral Diseases, National Clinical Research Center for Oral Diseases, Chinese Academy of Medical Sciences Research Unit of Oral Carcinogenesis and Management, West China Hospital of Stomatology, Sichuan University, Chengdu, Sichuan 610041, P. R. China. ${ }^{5}$ Stomatology Hospital, School of Stomatology, Cancer Center, Zhejiang University School of Medicine, and Key Laboratory of Oral Biomedical Research of Zhejiang Province, Clinical Research Center of Oral Diseases of Zhejiang Province, Hangzhou 310006 Zhejiang, China. ${ }^{6}$ Department of Health Sciences, The Graduate School of Dong-A University, Busan 49315, Republic of Korea. ${ }^{7}$ Department of Biomedical Sciences, Dong-A University, Busan 49315, Republic of Korea. ${ }^{8}$ MD Anderson UTHealth Graduate School of Biomedical Sciences, Houston 77030 TX, USA ${ }^{9}$ Department of Experimental Radiation Oncology, The University of Texas MD Anderson Cancer Center, Houston 77030 TX, USA. ${ }^{10}$ These authors

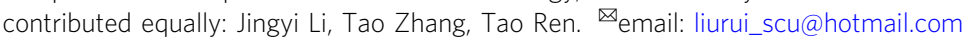


ypoxia is caused by insufficient supply of oxygen, which is a cofactor or substrate for various biological enzymatic reactions. Cells may gain tolerance in low oxygen environment, by either promoting angiogenesis to increase oxygen supply or enhancing mitochondria clearance to limit oxygen consumption $^{1,2}$. Hypoxia-inducible factor (HIF) act as a sensor to detect cellular oxygen levels, and binds to the hypoxia-responsive elements under hypoxia condition, thereby initiating the transcription of its target genes ${ }^{3}$. Oxygen supply in rapid growing tumors is frequently impaired as a result of disturbed microcirculation, inevitably leading to hypoxic stress in tumor cells ${ }^{4}$.

Autophagy is one of the cellular adaptive responses under multiple stresses, which enables intracellular materials delivered to lysosomes for degradation, and the products can be subsequently exported to the cytoplasm to be recycled ${ }^{5}$. As a highly conserved process in all eukaryotes, autophagy plays important roles in regulating cellular metabolism as well as tissue homeostasis by removing damaged macromolecules or even organelles $^{6,7}$. On the other hand, abnormal autophagy is closely associated with human diseases, including cancers ${ }^{8-11}$.

Autophagy is regulated by a complex molecular network. Atg1/ Unc-51 like autophagy activating kinase 1 (ULK1) is a key regulator of autophagy initiation ${ }^{12}$. At early steps during autophagy induction, active ULK1 regulates the recruitment of Atg14Lcontaining vacuolar sorting protein 34 (VPS34) complex ${ }^{13,14}$. ULK1 then phosphorylates Beclin 1, a binding partner for VPS34, thereby enhancing the activity of VPS34 complexes ${ }^{15-17}$. VPS34, the only class III phosphoinositide 3-kinase (PI3K) in mammals, phosphorylates phosphatidylinositol to produce phosphatidylinositol 3-phosphate (PI3P), which is necessary for phagophore membrane extension ${ }^{18}$. Finally, two ubiquitin-like conjugation systems promote lipidation of Microtubule-Associated Protein 1 Light Chain 3 (LC3), leading to cargo recognition and packaging in autophagosomes ${ }^{19-21}$. Though HIF1 $\alpha$ plays an important role in autophagy induction by releasing Beclin 1 from Bcl-2 22,23 , the mechanisms underlying autophagy initiation under hypoxic stress remains unclear.

In this study, we demonstrated that symmetrical dimethylation of ULK1 arginine (R)170, which is governed by PRMT5 and KDM5C, is sensitive to oxygen availability. This modification of ULK1 promotes autophagosome formation and mitochondria clearance, and is required for cell adaptation to hypoxic stress.

\section{Results}

Hypoxia-induced symmetrical dimethylation at ULK1 R170 is required for autophagy induction. To examine the effect of hypoxia on autophagy induction, we examined the levels of autophagy markers, LC3 II and p62, in LN229 human glioblastoma (GBM) cells, Huh7 hepatocellular carcinoma (HCC) cells, and human oral keratinocytes (HOKs) cultured in the hypoxic environment ( $1 \%$ oxygen). In all three cell lines, a dynamically increased LC3 II expression companied with a reduced p62 expression was detected (Fig. 1a). In addition, hypoxia resulted in increased puncta formation of LC3-GFP fusion protein in LN229 cells (Fig. 1b). These observations indicated that autophagy was efficiently activated in these cell lines. Hypoxia represses mTOR and activates AMPK, which are known as key upstream regulators of autophagy ${ }^{24,25}$. Deficiency of TSC1, which resulted in constant active mTOR and enhanced phosphorylation of mTOR substrate p70 Ribosomal S6 Kinase (S6k), or double knockout of AMPKa1a2, which caused declined phosphorylation of AMPK substrate ACC, did not affect hypoxiainduced accumulation of LC3 II or decrease of p62 (Supplementary Fig. 1a, b), suggesting that hypoxia-induced autophagy is independent of mTOR or AMPK.
ULK1 and ULK2 are pivotal players in autophagy initiation ${ }^{12}$. Knockout of ULK1 in mouse embryo fibroblasts (MEFs) reduced hypoxia-induced LC3 II accumulation and restored p62 expression (Fig. 1c), while no obvious effects were observed in ULK2 knockout MEFs (Supplementary Fig. 1c). This is consistent with previous reports that, in spite of some shared functions, ULK1 and ULK2 have distinct roles in autophagy induction ${ }^{22,26}$. AMPK and mTOR regulate autophagy through phosphorylating ULK1 ${ }^{22}$. However, a time-course experiment showed no obvious change in either mTOR-induced phosphorylation of ULK1 S757 or AMPKmediated phosphorylation of ULK1 S555 under 12 h-hypoxia treatment (Supplementary Fig. 1d), which could efficiently induce autophagy (Fig. 1a). ULK1 immunoprecipitates derived from hypoxia-stimulated LN229 cells was subjected to liquid chromatography-tandem mass spectrometry, leading to identification of a dimethylation at the evolutionally conserved ULK1 R170 (Supplementary Fig. 1e, f). Though a canonical 'RGG' motif is reported, arginine methylations were also found at various noncanonical sites ${ }^{27,28}$. Hypoxia-induced ULK1 R170 dimethylation was detected with a validated anti-symmetrically dimethylated R170 (R170me2s) antibody, but not anti-asymmetrically dimethylated R170 (R170me2a) antibody, in LN229, Huh7 or HOK cells (Fig. 1d, Supplementary Fig. 1g). The immunoblot signal could be abolished by incubation with a R170me2s, but not R170me, R170me2a or unmodified peptide (Supplementary Fig. 1h). Further, ULK1 R170me2s showed a dynamic response to oxygen availability in LN229 cells, and this modification was markedly increased when oxygen tension was decreased below 10\% (Fig. 1e). Though ULK1 and ULK2 share comparable protein molecular weight and similar protein sequence spanning ULK1 R170 and the corresponding ULK2 R163 (Supplementary Fig. 1i), the anti-ULK1 R170me2s antibody recognized immunoprecipitated Flag-ULK1, rather than Flag-ULK2, from hypoxiastimulated LN229 cells (Supplementary Fig. 1j). Consistently, only ULK1 deficiency totally blocked hypoxia-induced immunoblot signal using the anti-ULK1 R170me2s antibody (Supplementary Fig. 1k). These results suggested that hypoxic stress induces ULK1 R170me2s, while ULK2 was not methylated at the corresponding site R163.

To determine the effect of ULK1 R170me2s on hypoxiainduced autophagy, we depleted endogenous ULK1 expression by short hairpin RNA (shRNA), and established the reconstituted expression of triple-epitope S protein/FLAG/streptavidin-binding peptide (SFB)-tagged ULK1 R170lysine (K) mutant, which resists R170me2s, in LN229, Huh7 and HOK cells. Indeed, ULK1 R170K mutation substantially reduced hypoxia-induced accumulation of LC3 II, clearance of p62 (Fig. 1f) and formation of LC3-GFP puncta (Fig. 1g). Additionally, though loss of TSC1 or AMPKala2 showed negligible effects on the level of ULK1 R170me2s (Supplementary Fig. 1l, m), reconstituted expression of ULK1 R170K mutant largely attenuated autophagy induction in TSC1 or AMPKa1a2 knockout MEFs (Supplementary Fig. 1n, o). Further, we employed CRISPR/Cas9 genome-editing technology to knockin ULK1 R170K mutation in LN229 cells (Supplementary Fig. 2a). As expected, knockin expression of ULK1 R170K impeded autophagy induction in hypoxia-stimulated LN229 cells, as detected by immunoblot analyses of LC3 II and p62 expression, fluorescent analyses of LC3-GFP puncta and transmission electron microscope (TEM) analyses of the double-membraned autophagosome-like vacuoles (Fig. $1 \mathrm{~h}-\mathrm{j}$ ). Further, treatment of chloroquine (CQ) or analyses of LC3-RFP-GFP puncta revealed that ULK1 R170K mutation also blocked hypoxia-induced autophagic flux (Supplementary Fig. 2b-d). In contrast, ULK1 R170me2s was not obviously changed upon glucose or amino acids starvation, and ULK1 R170K mutation showed limited effects on autophagy induction in response to these stimuli 
a

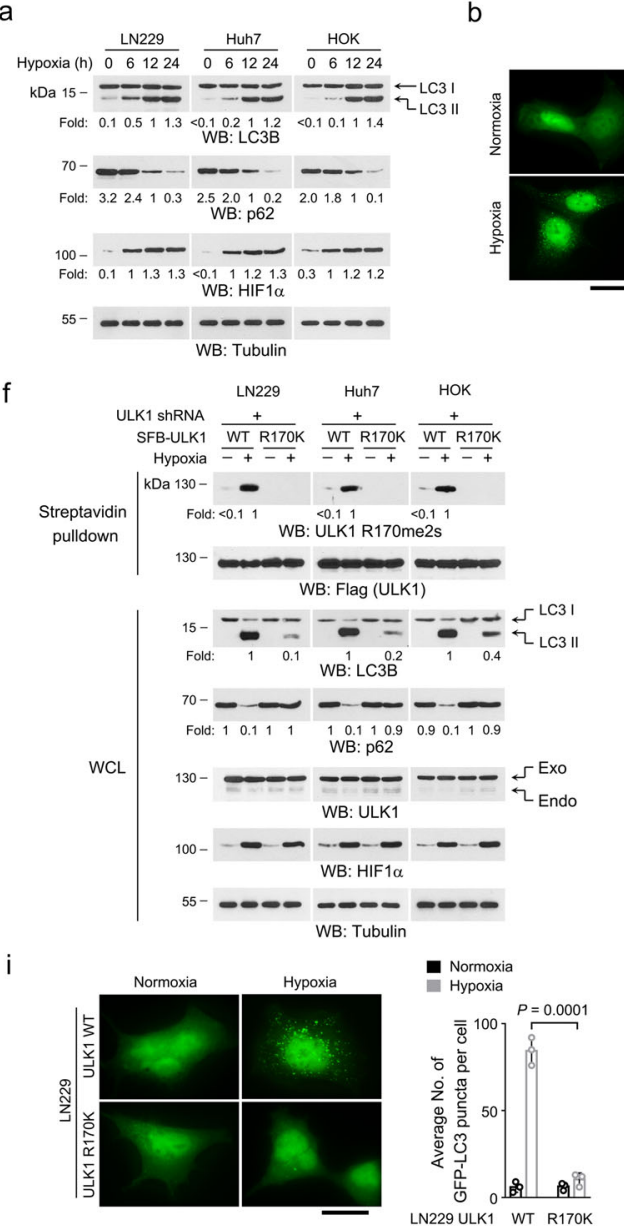

C

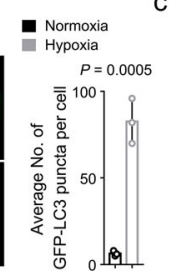

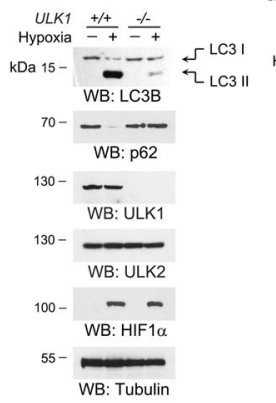

d
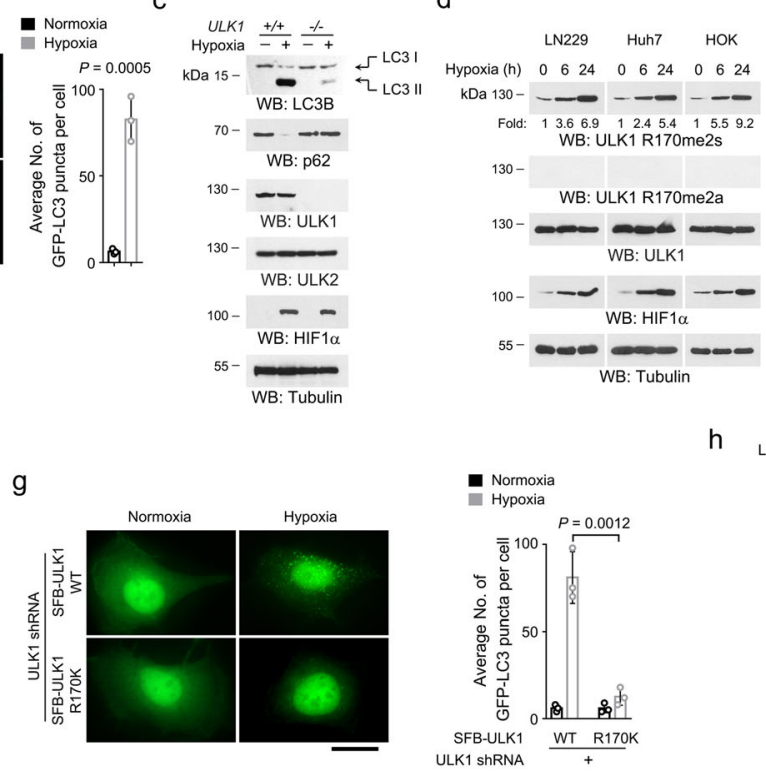

h

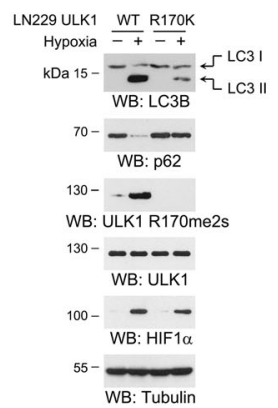

j

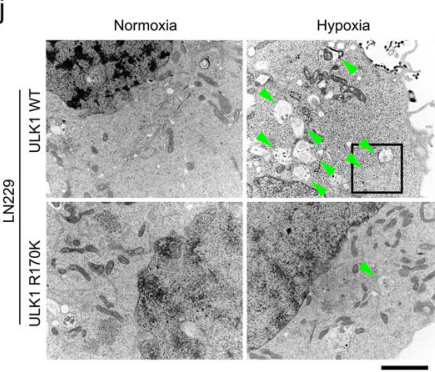

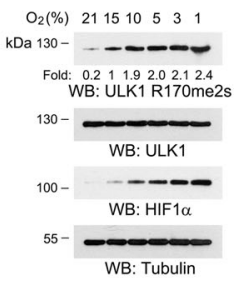

:ULK1 R170me2s

\section{.}
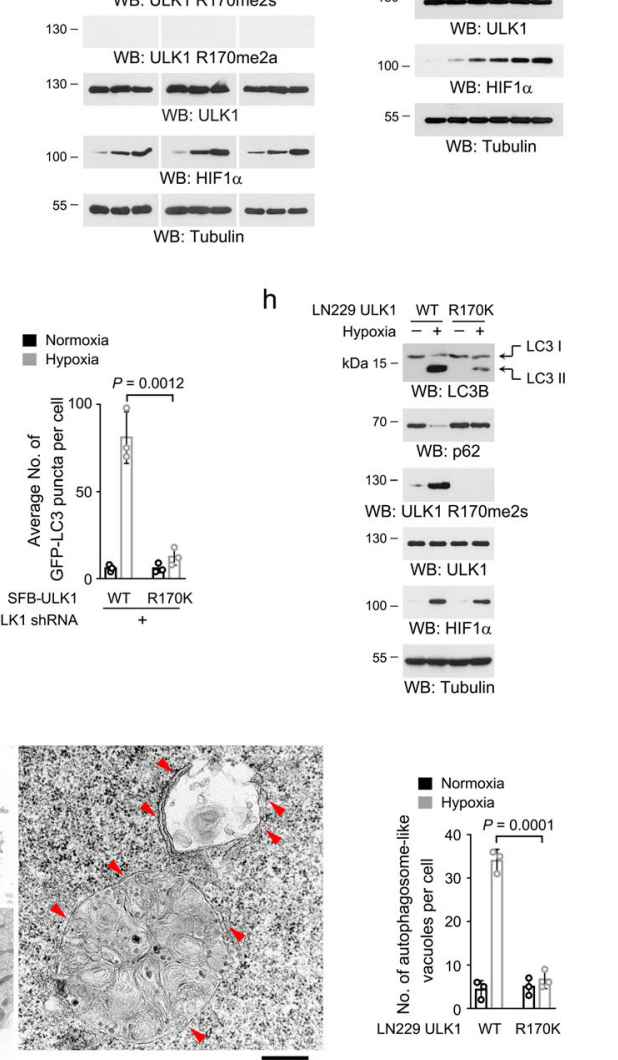

Fig. 1 ULK1 R170me2s is required for hypoxia-induced autophagy. a, c-f, h Immunoblot were performed with the indicated antibodies. a LN229, Huh7, and HOK cells were incubated with $1 \%$ oxygen. b LN229 cells expressed with LC3-GFP were incubated with $1 \%$ oxygen for $12 \mathrm{~h}$. LC3-GFP puncta were analyzed. Scale bar, $15 \mu \mathrm{m}$. Data represent the mean \pm SD from three independent experiments. $P$-value is from the two-sided $t$-test. c WT and ULK1-/MEFs were incubated with $1 \%$ oxygen for $12 \mathrm{~h}$. $\mathbf{d}$ LN229, Huh7, and HOK cells were incubated with $1 \%$ oxygen. e LN229 cells were incubated with indicated concentration of oxygen for $12 \mathrm{~h}$. $\mathbf{f}$ Endogenous ULK1-depleted LN229, Huh7, and HOK cells with reconstituted expression of WT SFB-ULK1 or SFB-ULK1 R170K were incubated with $1 \%$ oxygen for $12 \mathrm{~h}$. A streptavidin pulldown was performed. ULK1 shRNA targets non-coding region. Exo, exogenous; Endo, endogenous. $\mathbf{g}$ Endogenous ULK1-depleted LN229 cells with reconstituted expression of SFB-tagged WT ULK1 or SFB-ULK1 R170K were transfected with LC3-GFP plasmid. $48 \mathrm{~h}$ after transfection, cells were incubated with $1 \%$ oxygen for $12 \mathrm{~h}$. LC3-GFP puncta were analyzed. Scale bar, $10 \mu \mathrm{m}$. ULK1 shRNA targets non-coding region. Data represent the mean \pm SD from three independent experiments. $P$-value is from the two-sided $t$-test. $\mathbf{h}, \mathbf{j}$ Indicated cells were incubated with $1 \%$ oxygen for $12 \mathrm{~h}$. Immunoblot were performed (h). Ultrastructure was analyzed by TEM (j). The boxed area was enlarged. Green arrowheads indicate autophagosome-like vacuoles, and red arrowheads indicate double membrane structures. Scale bar for original images, $2 \mu$ m; scale bar for enlarged images, $400 \mathrm{~nm}$. Data represent the mean \pm SD from three independent experiments. $P$-value is from the two-sided $t$-test. $\mathbf{i}$ WT LN229 cells and LN229 cells with knockin expression of ULK1 R170K were transfected with LC3-GFP plasmid. $48 \mathrm{~h}$ after transfection, cells were incubated with $1 \%$ oxygen for $12 \mathrm{~h}$. LC3-GFP puncta were analyzed. Data represent the mean \pm SD from three independent experiments. $P$-value is from the two-sided $t$-test. Scale bar, $10 \mu \mathrm{m}$. Source data are provided as a Source data file.

(Supplementary Fig. 2e, f). These results strongly suggest that hypoxia-induced ULK1 R170me2s activates autophagy in an mTOR or AMPK-independent manner.

ULK1 R170 is symmetrically dimethylated by PRMT5 and demethylated by KDM5C. Generation of asymmetric dimethylarginines is catalyzed by type I protein arginine methyltransferases (PRMTs), while type II enzymes, PRTM5 and PRMT7, catalyzes symmetric arginine dimethylation ${ }^{29}$. ShRNAmediated knockdown of PRMT5 or PRMT7 revealed that PRMT5, rather than PRMT7, was involved in ULK1 R170me2s (Fig. 2a, Supplementary Fig. 3a). As expected, association between endogenous ULK1 and PRMT5 was detected in LN229, Huh7 and HOK cells cultured in both normoxia or hypoxia conditions (Fig. 2b); in contrast, ULK2 was not detected in PRMT5 immunoprecipitates (Fig. 2b). We next conducted an in vitro methylation analysis by incubating purified WT Flag-PRMT5 or catalytic-dead Flag-PRMT5 R368A mutant ${ }^{30}$ protein with WT His-ULK1 or His-ULK1 R170K protein, in the presence of S-adenosylmethionine as a methyl group donor. We found that WT PRMT5, but not PRMT5 R368A mutant, symmetrically dimethylated His-ULK1, which could be blocked by R170K mutation (Fig. 2c).

We next asked whether hypoxia enhanced PRMT5-mediated ULK1 R170me2s. Nevertheless, interaction between PRMT5 and ULK1 was not altered in hypoxia-treated LN229 cells (Supplementary Fig. 3b). An in vitro methylation analysis showed that oxygen concentration was not a determinant factor for PRMT5 to methylate ULK1 R170 (Supplementary Fig. 3c). Further, depletion of HIF1a had no obvious effect on hypoxia-mediated ULK1 
a

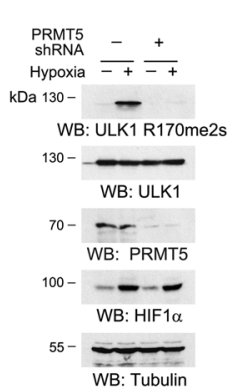

e

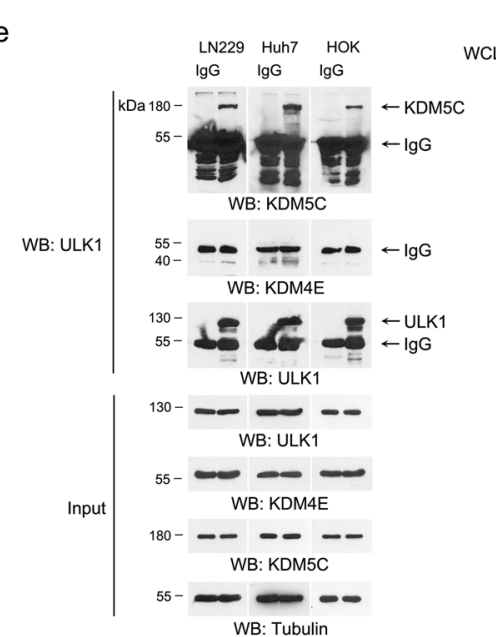

b

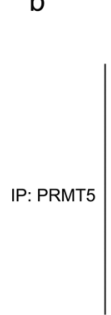

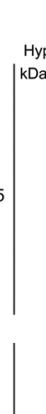$$
{ }_{\lg }^{\mathrm{LN} 229}{ }_{\operatorname{lgG}}^{\mathrm{Huh}}{ }_{\operatorname{lgg}}^{\text {HOK }}
$$

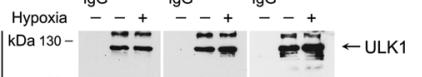$$
55-\infty-\infty \operatorname{Cog}
$$$$
{ }_{55-}^{130-}-\infty \mathrm{WBG}
$$

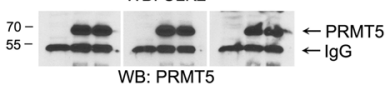$$
130--\infty----\square
$$
WB: ULK1$$
130-\ldots-\cdots \infty
$$$$
\text { WB: ULK2 }
$$$$
\text { 70- - - }-\infty
$$$$
\text { WB: PRMT5 }
$$$$
\text { 55- }=05
$$$$
\text { WB: Tubulin }
$$$$
\text { f }
$$

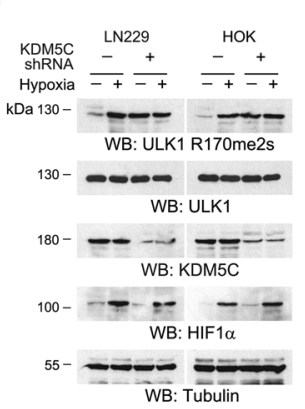

g c

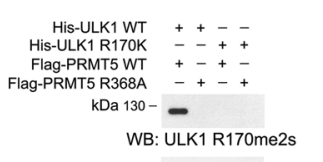

$130-$

WB: ULK1 R170me2a

130 - $\underset{W B: U K_{1}}{\longrightarrow}$

WB: ULK1

$70--2-\infty$

WB: PRMT5 d

$$
\begin{aligned}
& \text { IOx1 } \frac{-+}{-}++ \\
& \text { Kypoxia } \\
& \text { KDa 130- ULK1 R170me2s } \\
& \text { W30- WB: ULK1 } \\
& \text { WB: HIF1a } \\
& \text { WB: Tubulin }
\end{aligned}
$$

h

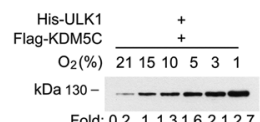

Fold: 0.211 .31 .62 .12 .7

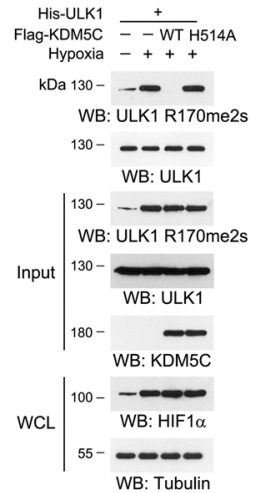

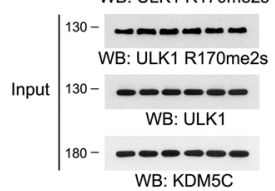

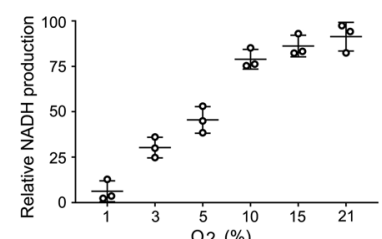

Fig. 2 ULK1 R170 is symmetrically dimethylated by PRMT5 and demethylated by KDM5C. a-h Immunoblot were performed with the indicated antibodies. a LN229 cells with or without expression of PRMT5 shRNA were incubated with 1\% oxygen for 12 h. b LN229, Huh7 or HOK cells were incubated with $1 \%$ oxygen for $12 \mathrm{~h}$. Immunoprecipitations were performed using an anti-PRMT5 antibody. c Purified WT His-ULK1 or His-ULK1 R170K protein was mixed with purified WT Flag-PRMT5 or Flag-PRMT5 R368A protein for an in vitro methylation assay. d LN229 cells were pretreated with or without $200 \mathrm{mM}$ IOX1 for $12 \mathrm{~h}$, followed by incubation under $1 \%$ oxygen for $12 \mathrm{~h}$. e LN229, Huh7 or HOK cells were cultured in normoxia condition. Immunoprecipitations were performed using an anti-ULK1 antibody. f LN229 and HOK cells with or without expression of KDM5C shRNA were incubated with $1 \%$ oxygen for $12 \mathrm{~h} . \mathbf{g}$ LN229 with expression of His-ULK1 were incubated under $1 \%$ oxygen for $12 \mathrm{~h}$. His-ULK1 proteins were pulldown, the precipitates were mixed with purified WT Flag-KDM5C or Flag-KDM5C H514A mutant protein for an in vitro demethylation assay in normoxia condition. $\mathbf{h}$ LN229 with expression of His-ULK1 were incubated under $1 \%$ oxygen for $12 \mathrm{~h}$. His-ULK1 proteins were pulldown, washed and mixed with purified WT Flag-KDM5C protein for an in vitro demethylation assay in presence of indicated concentrations of oxygen. i Purified Flag-KDM5C protein was mixed with ULK1 $\mathrm{R} 170 \mathrm{me} 2 \mathrm{~s}$ peptide for an in vitro demethylation assay in presence of indicated concentrations of oxygen. Data represent the mean \pm SD from three independent experiments. Source data are provided as a Source data file.

R170me2s (Supplementary Fig. 3d). These results suggested that, though PRMT5 catalyzes ULK1 R170me2s, hypoxia-induced ULK1 R170me2s was not caused by PRMT5 alteration and was independent of HIFa.

To find out whether hypoxia-induced ULK1 R170me2s was governed by a demethylase, LN229 cells were treated with 5carboxy-8-hydroxyquinoline (IOX1), a pan-inhibitor of the 2oxoglutarate-dependent JMJD family demethylase ${ }^{31}$. Strikingly, though IOX1 markedly enhanced ULK1 R170me2s under normoxia condition, hypoxia treatment could not further increase ULK1 R170me2s (Fig. 2d), suggesting that a demethylase was probably involved. KDM4E and KDM5C, belonging to the KDM4 and KDM5 lysine demethylase subfamilies, were recently evidenced to be capable to catalyze de-dimethylation of arginine on histone peptides (Supplementary Fig. 3e) ${ }^{32}$. Notably, interaction between endogenous ULK1 and KDM5C, but not KDM4E, was confirmed by immunoprecipitation in LN229, Huh7 and HOK cells (Fig. 2e).

To determine whether KDM5C was capable to regulates ULK1 R170me2s, we knockdown the expression of KDM5C by two distinct shRNAs, and found a markedly enhanced ULK1 R170me2s and autophagy induction in normoxia condition (Supplementary Fig. 3f-h). However, this effect was not further augmented by hypoxia stimulation (Fig. 2f). In contrast, overexpression of exogenous KDM5C only limitedly reduced ULK1 R170me2s in hypoxia condition (Supplementary Fig. 3i). Further, we mixed His-tagged ULK1 protein purified from hypoxia-stimulated LN229 cells with purified WT Flag-KDM5C protein or catalytic-dead Flag-KDM5C H514A protein ${ }^{33}$, and found that only WT Flag-KDM5C could remove ULK1 R170me2s (Fig. 2g).

To decipher how hypoxia affects KDM5C-dependent demethylation of ULK1 R170me2s, we initially tested whether hypoxia regulates $\mathrm{KDM} 5 \mathrm{C}$ expression. However, only a slight increase in KDM5C expression could be detected in LN229, Huh7 and HOK cells after $24 \mathrm{~h}$ hypoxia treatment (Supplementary Fig. 3j), while the expression of PRMT5 and ULK1 was almost unchanged (Supplementary Fig. 3j). In addition, nickle-nitrilotriacetic acidaided (Ni-NTA) pull down assay demonstrated that equal amount of KDM5C protein could be pulled down by Histagged ULK1 under normoxia and hypoxia conditions (Supplementary Fig. 3k). KDM5C catalyzes demethylation through an oxidative reaction that requires a-ketoglutarate $(\alpha \mathrm{KG})$ and oxygen as cofactors ${ }^{34}$. Treatment with various dosages of membrane-permeable dimethyl (DM)-aKG did not alter ULK1 R170me2s under hypoxia (Supplementary Fig. 31), suggesting that 
hypoxia-regulated $\mathrm{KDM} 5 \mathrm{C}$ is not due to $\mathrm{aKG}$ deficiency. To determine whether $\mathrm{KDM} 5 \mathrm{C}$ activity was regulated by oxygen availability, purified KDM5C protein was mixed with R170dimethylated ULK1 protein, purified from hypoxia-stimulated LN229 cells, for an in vitro demethylation assay under different oxygen concentrations. We found that ULK1 R170me2s was dynamically increased as oxygen concentration decreased (Fig. 2h). Further, impeded KDM5C activity was also observed by using the ULK1 R170me2s peptide as a substrate (Fig. 2i); a rapid change of $\mathrm{KDM} 5 \mathrm{C}$ activity was found when oxygen tension was reduced below $10 \%$ (Fig. 2i), which was consistent with cellular ULK1 R170me2s status (Fig. 1e). These results suggested that KDM5C activity is sensitive to oxygen availability, and hypoxia-induced ULK1 R170me2s is due to repressed KDM5Cmediated demethylation.

R170me2s activates ULK1 by facilitating ULK1 T180 autophosphorylation. We therefore determined whether ULK1 R170me2s affect ULK1 activity under hypoxia. As expected, hypoxia treatment induced ULK1 activity in LN229, Huh7 and HOK cells, while only a limited increase was found in ULK1 R170K mutant (Fig. 3a, Supplementary Fig. 4a). Depletion of endogenous ULK1 and reconstituted expression of ULK1 R170K or a kinase dead ULK1 K46I mutant ${ }^{35}$, but not WT ULK1, substantially abolished ULK1-dependent phosphorylation of Atg13 S355 $5^{36}$ and Beclin $1 \mathrm{~S} 15^{37}$ in hypoxia-stimulated LN229, Huh7 and HOK cells (Fig. 3b, Supplementary Fig. 4b). Phosphorylation of GST-Atg13 or His-Beclin 1 by purified WT ULK1 protein, rather than ULK1 R170K mutant protein, was largely enhanced by PRMT5-mediated ULK1 R170me2s (Fig. 3c). Consistently, incubation with WT KDM5C protein inhibited R170me2s-bearing ULK1 protein, which was purified from hypoxia-stimulated LN229 cells, to phosphorylate GST-Atg13 or His-Beclin 1; while this effect was abolished by the catalytic-dead KDM5C H514A mutation (Fig. 3d). Further, knockin expression of ULK1 R170K in LN229 cells or reconstituted expression this mutant in Huh7 or HOK cells eliminated hypoxia-induced activity vacuolar protein sorting 34 (VPS34) in Atg14L immunoprecipitates (Fig. 3e, Supplementary Fig. 4c) without affecting the expression of Beclin 1 and VPS34, and abrogated the formation of EGFP-FYVE puncta (Fig. 3f). These data suggested that ULK1 R170me2s is required for ULK1 activation under hypoxia condition.

A structure analysis (PDB code: $4 \mathrm{WNP}$ ) revealed that ULK1 $\mathrm{R} 170$ is located within a conserved loop domain (Supplementary Fig. 4d), and is spatially closed to T180 (Fig. 3g). Autophosphorylation at ULK1 T180 under stress conditions, such as rapamycin treatment, is required for ULK1 activation ${ }^{38}$. Indeed, immunoblot with a validated specific antibody (Supplementary Fig. 4e) showed a much higher level of T180 phosphorylation under hypoxia treatment (Fig. 3h). Interestingly, glucose or amino acids deprivation also induced similar phosphorylation of ULK1 T180 (Supplementary Fig. 4f). Depletion of endogenous ULK1 and reconstituted expression of a non-phosphoryable ULK1 T180A mutant alleviated hypoxia-stimulated phosphorylation of Atg13 and Beclin 1, activation of VPS34, accumulation of LC3 II, clearance of p62 and formation of LC3-GFP puncta, though no obvious differences was found under normoxia condition (Supplementary Fig. 4g-i).

We, therefore, determined whether ULK1 R170me2s is required for T180 autophosphorylation. Indeed, hypoxiainduced ULK1 T180 phosphorylation was substantially impaired by knockin expression of ULK1 R170K mutation (Fig. 3i). An in vitro kinase assay showed that PRMT5-mediated ULK1 R170me2s was companied with a markedly increased T180 phosphorylation when ULK1 protein was incubated with ATP (Fig. 3j), and this effect was eliminated by ULK1 R170K mutation (Fig. 3k). Consistently, knockdown of PRMT5 in LN229, Huh7, and HOK cells largely abolished hypoxia-induced ULK1 T180 phosphorylation, phosphorylation of Atg13 and Beclin 1, LC3 II accumulation, p62 degradation, and FYVE and LC3 puncta formation (Supplementary Fig. 5a-c). In contrast, transfection of pcDNA3-KDM5C construct, which increased cellular KDM5C expression by $2-4$ folds, only limitedly affected these hypoxiainduced effects (Supplementary Fig. 5d-f). Together, these data suggested that hypoxia-repressed KDM5C causes ULK1 R170me2s, and subsequent T180 autophosphorylationdependent ULK1 activation, which is required for hypoxiainduced autophagy induction.

ULK1 R170me2s promotes mitochondria turnover and promotes cell growth. Hypoxia induces mitochondria fission, thereby facilitating mitochondrial turnover by autophagy to limit cellular oxygen consumption ${ }^{39,40}$. Immunofluorescent analyses of Tomm20, a mitochondrial marker ${ }^{41}$, revealed that hypoxia treatment induced comparable mitochondrial fragmentation in LN229 cells with either WT ULK1 or ULK1 R170K mutant (Fig. 4a). Notably, ULK1 R170K mutation largely restored the Tomm20-positive mitochondrial area in hypoxia-stimulated cells (Fig. 4a), hinting that ULK1 R170me2s probably facilitates the degradation of mitochondria. Indeed, hypoxia treatment markedly enhanced the engulfment of mitochondria in autophagosome-like vacuoles in WT LN229 cells shown by TEMbased ultrastructure analyses, which was substantially abolished by ULK1 R170K mutation (Fig. 4b). Further, ULK1 R170K mutation or knockdown of PRMT5 partially rescued the loss of mitochondrial DNA (mtDNA) in hypoxia-stimulated LN229, Huh7, and HOK cells, suggesting an impaired mitochondrial turnover (Fig. 4c, Supplementary Fig. 6a, b). Given that mitochondria are the major oxygen-consuming organelles for mammalian cells ${ }^{42}$, we incubated cells with $1 \%$ oxygen for $18 \mathrm{~h}$, and measured the cellular oxygen consumption rate (OCR) immediately in normoxia condition. As expected, hypoxia treatment resulted in about 70\% decrease in cellular OCR in LN229, Huh7 and HOK cells (Fig. 4d, Supplementary Fig. 6c, d). In contrast, either ULK1 R170K mutation or PRMT5 depletion restored cellular OCR, though it did not apparently alter cellular OCR without hypoxia stimulation (Fig. 4d, Supplementary Fig. 6c, d). However, the level of mtDNA or cellular OCR was not obviously changed upon overexpression of KDM5C (Supplementary Fig. 6e, f). In line with this, BrdU incorporation assay showed that mutation of ULK1 R170K or reconstituted expression of Atg13 S355A/Beclin 1 S15A further reduced hypoxia-repressed proliferation (Fig. 4e, Supplementary Fig. 6g, h). In addition, an increased cell death was also observed in ULK1-mutated LN229 cells upon hypoxia treatment by trypan blue exclusion assay (Fig. 4f). These results suggested that ULK1 R170me2s plays a crucial role in mitochondrial turnover and maintains cell viability in response to hypoxia stress.

To find out the impact of ULK1 R170me2s on tumor development, we intracranially injected athymic nude mice with WT LN229 cells or LN229 cells with knockin expression ULK1 R170K. Compared to WT LN229 cells, ULK1 R170K mutation inhibited tumor growth (Fig. 4g) with decreased cell proliferation (Fig. 4h) and elevated cell death (Supplementary Fig. 6i). Immunohistochemical staining with validated anti-ULK1 R170me2s or anti-ULK1 pT180 antibody (Supplementary Fig. 6j, k) revealed that ULK1 R170K mutation abolished ULK1 R170me2s, and reduced the phosphorylation of ULK1 T180, Atg13 S355, and Beclin 1 S15 in the tumor samples derived from 
a
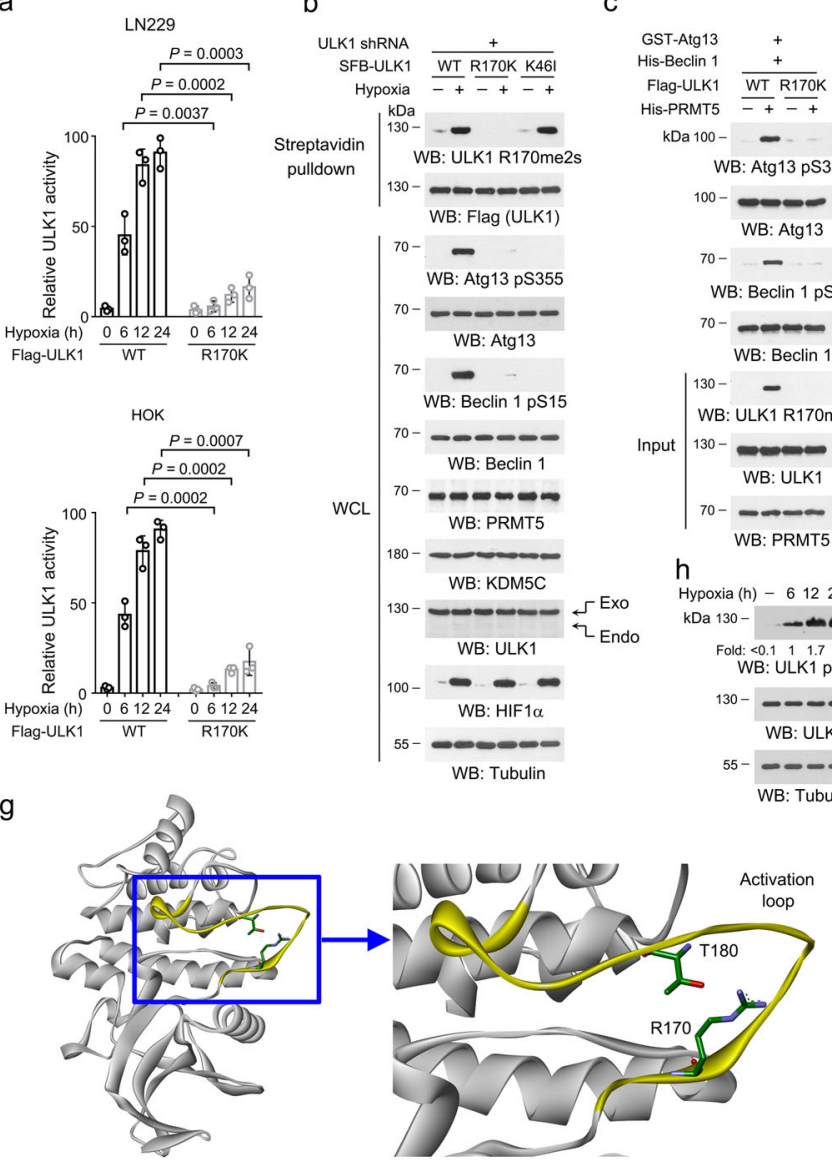

c

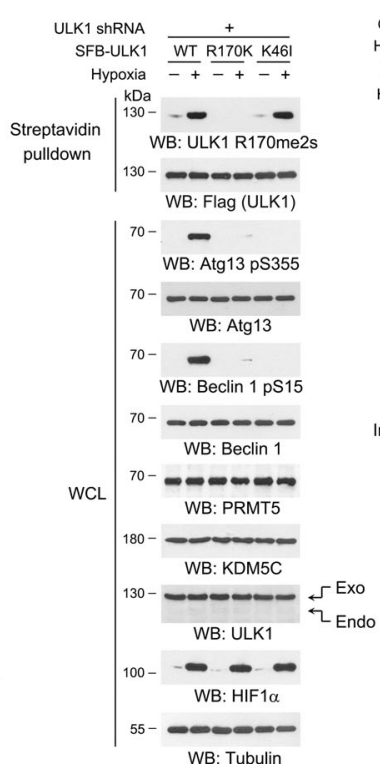

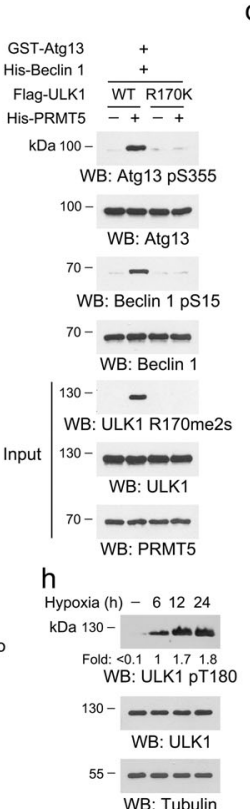

d
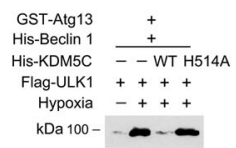

WB: Atg13 pS355

100

WB: Atg13

$70-\ldots$

WB: Beclin 1 pS15

70 -

WB: Beclin 1

Input $\mid \begin{aligned} & \text { 130- } \\ & \text { WB: ULK1 R170me2s }\end{aligned}$

Input ${ }_{130-}^{130-}$

\begin{tabular}{l|l} 
IP: & $130-$ \\
Flag & WB: ULK1 R170me2s
\end{tabular}

Flag

130 - D-O -

WCL $\mid \begin{aligned} & 100- \\ & \text { WB: HIF } 1 \alpha \\ & \text { WB: Tubulin }\end{aligned}$

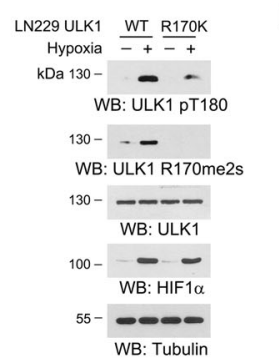

- Normoxia

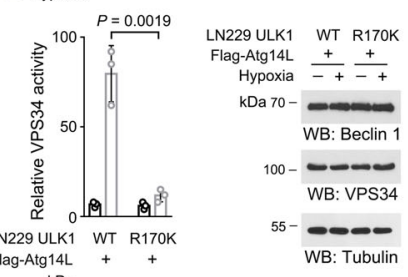

Flag-Atg14L

IP. Flag $\mid \begin{aligned} & \mathrm{kDa} \\ & 70-\end{aligned}$

WB: Flag (Atg14L)

f

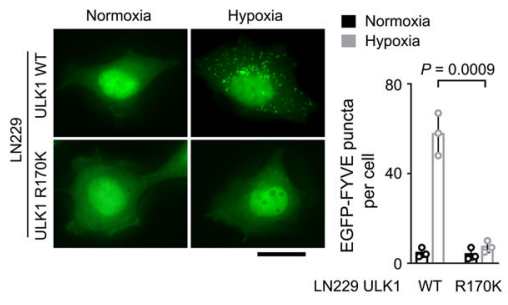

k

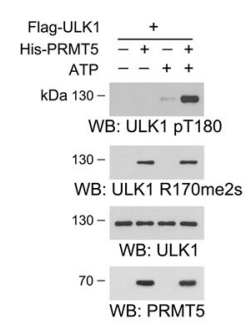

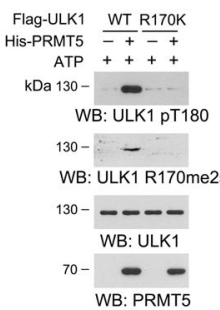

Fig. 3 R170me2s activates ULK1 by facilitating ULK1 T180 autophosphorylation. b-e, h-k Immunoblot were performed with the indicated antibodies. a Indicated cells were incubated under $1 \%$ oxygen for $12 \mathrm{~h}$. Flag-ULK1 proteins were precipitated, and ULK1 kinase activity was analyzed. Data represent the mean \pm SD from three independent experiments. $P$-values are from the two-sided $t$-tests. Bonferroni correction was used for multiple hypothesis correction. b Indicated LN229 cells incubated under 1\% oxygen for $12 \mathrm{~h}$. Exo, exogenous; Endo, endogenous. c Purified ULK1 proteins was immobilized on beads, and mixed with purified His-PRMT5 protein for an in vitro methylation assay. Immobilized ULK1 proteins were washed, and mixed with purified GST-Atg13 or His-Beclin 1 proteins for an in vitro kinase assay. d LN229 cells with expression of Flag-ULK1 were incubated under $1 \%$ oxygen for $12 \mathrm{~h}$. Flag-ULK1 proteins were pulldown, washed and mixed with purified WT His-KDM5C or His-KDM5C H514A protein for an in vitro demethylation assay under normoxia condition. The ULK1 precipitates were washed, and mixed with purified GST-Atg13 or His-Beclin 1 proteins for an in vitro kinase assay. e Indicated cells were incubated under $1 \%$ oxygen for $12 \mathrm{~h}$. VPS34 activity in the precipitates were measured. Data represent the mean \pm SD from three independent experiments. $P$-value is from the two-sided $t$-test. $\mathbf{f}$ Indicated cells were incubated under $1 \%$ oxygen for $12 \mathrm{~h}$. EGFP-FYVE puncta formation was analyzed. Scale bar, $6 \mu \mathrm{m}$. Data represent the mean \pm SD from three independent experiments. $P$-value is from the two-sided t-test. $\mathbf{g}$ Human ULK1 structure (PDB code: 4WNP) shows an activation loop domain (yellow). The surrounding areas of R170 and T180 are boxed and enlarged. For the side chain of R170 and T180, nitrogen, oxygen and carbon atoms were shown in purple, red and green, respectively. $\mathbf{h}$ LN229 cells were incubated under $1 \%$ oxygen. $\mathbf{i}$ Indicated cells were incubated under $1 \%$ oxygen for $12 \mathrm{~h}$. j, $\mathbf{k}$ Purified WT Flag-ULK1 (j) or/and Flag-ULK1 R170K (k) proteins were subjected to a His-PRMT5 proteinmediated in vitro methylation assay and an in vitro autophosphorylation assay. Source data are provided as a Source data file.

the mice in normoxia condition (Supplementary Fig. 61). Notably, exposure of the tumor-bearing mice to $11 \%$ low oxygen environment substantially impaired tumor growth (Fig. 4g). Such low oxygen availability resulted in elevated expression of hypoxia markers HIF1 $\alpha$ and BNIP ${ }^{43,44}$, increased ULK1 R170me2s, as well as enhanced phosphorylation of ULK1 T180, Atg13 S355, and Beclin 1 S15 (Supplementary Fig. 6l). Notably, expression of ULK1 R170K mutation attenuated low-oxygen-induced phosphorylation of ULK1 T180, Atg13 S355 and Beclin 1 S15, and accordingly further curbed tumor growth (Fig. $4 \mathrm{~g}$ ) with repressed cell proliferation (Fig. 4h) and increased cell death (Supplementary Fig. 6i). To further expand our findings, we injected fluorescent probe-labeled HOK cells into the pericardial cavity of zebrafish embryos (Supplementary Fig. $6 \mathrm{~m})^{45}$. As expected, ULK1 R170K mutation further reduced the number of cells in zebrafish that were maintained under hypoxia condition
(Supplementary Fig. 6n). These results suggested that ULK1 R170me2s copes with hypoxia stress to maintain cell viability.

To evaluate the clinical relevance of ULK1 R170me2s, human glioblastoma samples was analyzed by immunohistochemical staining. We show that the level of HIF1a, BNIP3, ULK1 R170me2s, ULK1 pT180, and Beclin 1 pS15 were positively corelated (Fig. 4i, Supplementary Fig. 6o). Quantification of staining intensities revealed that these correlations were significant (Fig. 4j, Supplementary Fig. 6p). Notably, a significantly shorter survival time was found in those GBM patients with a high level of R170me2s (Fig. 4k). These results underscore the clinical relevance of ULK1 R170 me2s in tumor development.

\section{Discussion}

Oxygen concentration is important for tissue homeostasis. As a result of low oxygen availability, the growth and viability of cells 
a

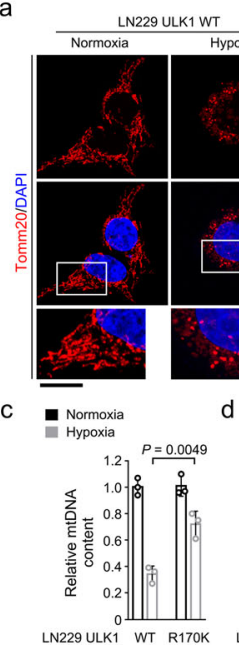

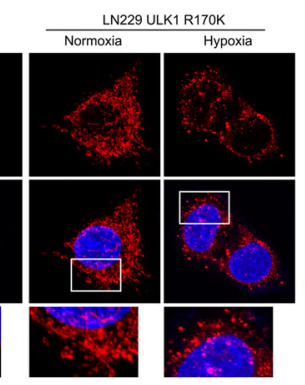

d. Untreated

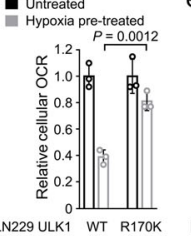

e normoxia

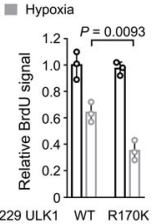

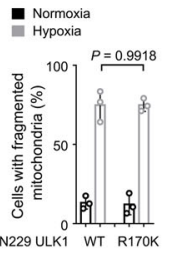

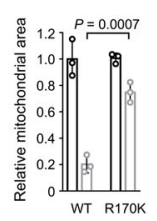

f
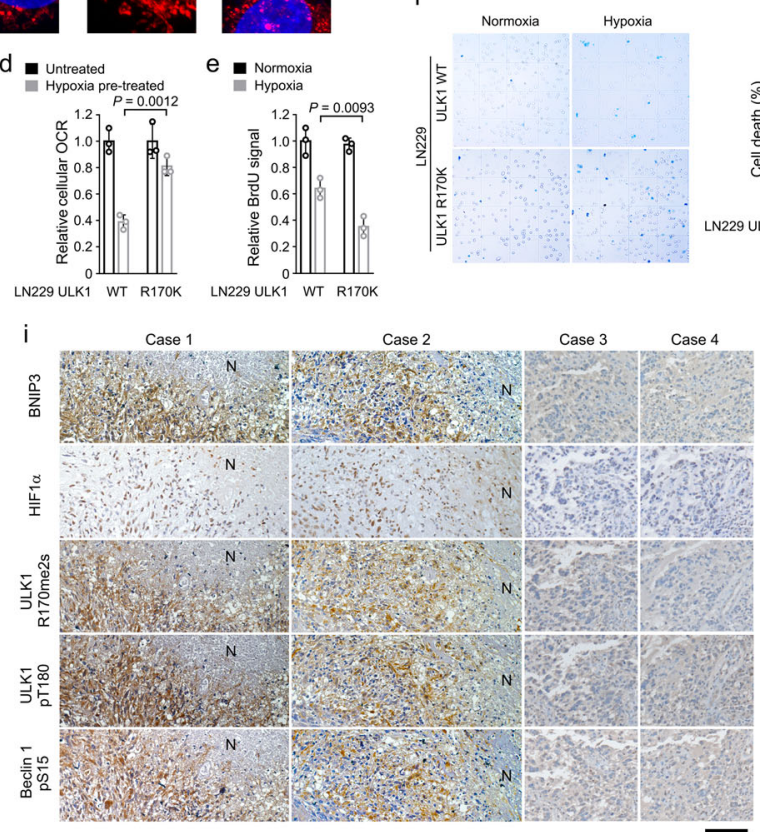

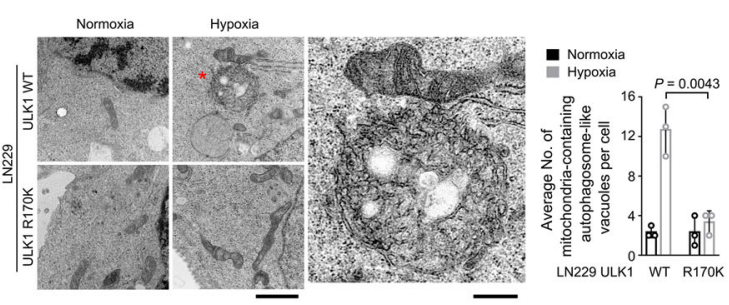

g
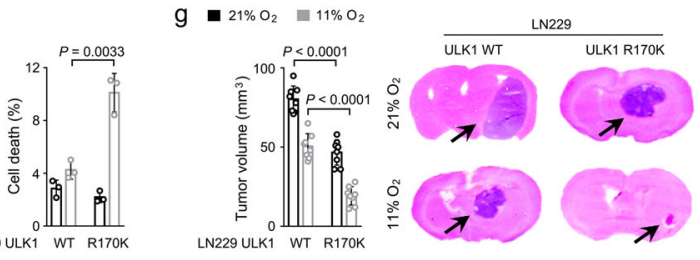

LN229 ULK1 WT R170K
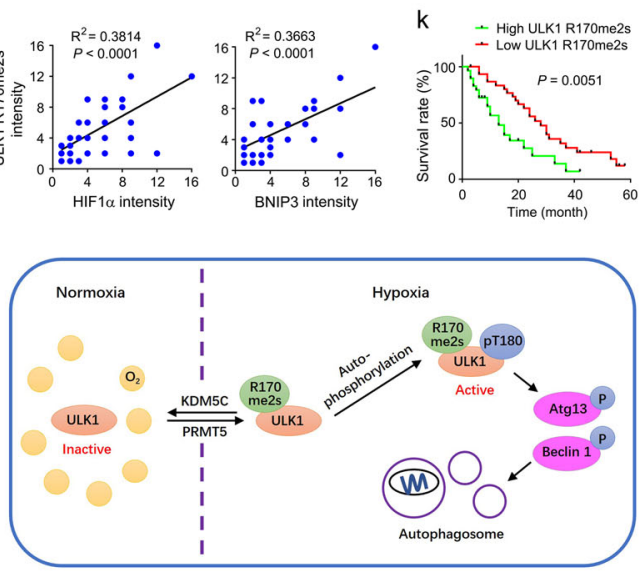

Fig. 4 ULK1 R170me2s promotes mitochondria turnover and tumor growth under hypoxia. c-e Data was normalized to the WT untreated group. a, b Indicated cells were incubated under $1 \%$ oxygen for $12 \mathrm{~h}$. Immunofluorescent staining was performed. The boxed areas (Scale bar, $10 \mu \mathrm{m}$ ) were enlarged (Scale bar, $4 \mu \mathrm{m}$ ) (a). Ultrastructure was analyzed. Vacuoles (Scale bar, $1.5 \mu \mathrm{m}$ ) labeled with asterisk were enlarged (Scale bar, $500 \mathrm{~nm}$ ) (b). Data represent the mean \pm SD from three independent experiments. $P$-value is from the two-sided $t$-test. $\mathbf{c}$ Indicated cells were incubated under $1 \%$ oxygen for $24 \mathrm{~h}$. The level of mitochondrial DNA (mtDNA) was measured. Data represent the mean \pm SD from three independent experiments. $P$-value is from the two-sided $t$-test. $\mathbf{d}$ Indicated cells were stimulated with $1 \%$ oxygen for $18 \mathrm{~h}$, and the cellular OCR was measured immediately in normoxia condition. Data represent the mean \pm SD from three independent experiments. $P$-value is from the two-sided $t$-test. e, $\mathbf{f}$ Indicated cells were incubated under $1 \%$ oxygen for $48 \mathrm{~h}$. BrdU incorporation assay (e) and Trypan blue exclusion assay (f) were performed. Data represent the mean \pm SD from three independent experiments. $P$-values are from the two-sided $t$-tests. $\mathbf{g}$ Mice $(n=8)$ were intracranially injected with indicated LN229 cells, and were maintained in ambient atmosphere or $11 \%$ oxygen tension before sacrificed for examination of tumor growth (left panel). Representative H\&E-stained coronal brain sections are shown (right panel). Data represent the mean \pm SD. P-values are from the two-sided t-tests. Bonferroni correction was used for multiple hypothesis correction. $\mathbf{h}$ Immunochemical staining of mice tumors. Scale bar, $75 \mu \mathrm{m}$. i Immunochemical staining of human GBM tissues. Scale bar, $100 \mu \mathrm{m}$. $\mathrm{N}$, necrotic region. $\mathbf{j}$ Quantification of the immunochemical staining of human GBM samples $(n=40)$. $\mathbf{k}$ Kaplan-Meier plots of the overall survival time of patients $(n=60)$ with distinct ULK1 R170me2s levels. P-value is from the two-sided log-rank test. I Model of dynamic ULK1 methylation and autophagy induction under hypoxia. Source data are provided as a Source data file.

is reduced ${ }^{46}$. Autophagy is one of the activated cellular responses under multiple stressful conditions, including hypoxia ${ }^{5}$. Nevertheless, how the early steps during autophagy induction are regulated in a hypoxic environment was relatively unknown. In present study, we show that oxygen-sensitive dimethylation of ULK1 R170 is accumulated under hypoxia, which facilitates activation of ULK1, phosphorylation of ULK1 substrates Atg13 and Beclin 1, and subsequent formation of autophagosomes. Autophagy-dependent clearance of mitochondria reduced oxygen consumption, render cells resistant to low oxygen tension, and maintain cell viability (Fig. 4l).

Arginine methylation is a prevalent post-translational modification found on both nuclear and cytoplasmic proteins. This modification enables covalent addition of methyl groups to the side chain of arginine residues in a protein, predominantly in three types in eukaryotic cells: mono, symmetric and asymmetric.
Methylation and demethylation of arginine residues is a dynamic cyclical process, and a well-balanced arginine methylation is required for cell proliferation and survival ${ }^{47-49}$. In the present data, we demonstrate that ULK1 R170me2s status was dynamically controlled by a methylation/demethylation system, which consist of PRMT5 and KDM5C. PRMT5, formerly termed Janus kinase-binding protein 1, that catalyzes the formation of monomethylarginine and symmetric dimethylarginine in a variety of proteins ${ }^{50}$. In contrast, KDM5C is a member of 2-oxoglutaratedependent JmjC methylysine demethylases, which has been recently evidenced as an arginine demethylase ${ }^{32}$. Our data show that ULK1 could be methylated by PRMT5 at R170, which is insusceptible to oxygen concentration. Notably, ULK1 R170me2s was largely accumulated under hypoxia condition due to the repressed-KDM5C, which use oxygen as a cofactor to catalyze arginine demethylation through an oxidation reaction. 
It is widely accepted that both mTOR and AMPK regulate ULK1 activity in different ways. When sufficient nutrients exist, mTOR stays active, which suppressed autophagy initiation by phosphorylating and blunting ULK $1^{51}$. In response to glucose deprivation, AMPK is activated in case of low ATP levels, and in turn activates ULK1 by releasing it from $\mathrm{mTOR}^{22}$. Though repressed MTOR and active AMPK was previously detected under hypoxia condition, our results show that accumulation of LC3 II and formation of LC3-GFP puncta occurs in prior to mTOR or AMPK-mediated ULK1 phosphorylation, which suggested that mTOR or AMPK was not likely the major regulator for hypoxia-induced autophagy.

HIF1 $\alpha$ plays a crucial role in the regulation of oxygen homeostasis. HIFla is hydroxylated at proline 402/564 under normoxia condition, which could be recognized by von Hippel Lindau protein, leading to the recruitment of ubiquitin protein ligase complexes that target HIF1 $\alpha$ for proteasomal degradation. Under hypoxic conditions, HIFla hydroxylation is largely abolished, since oxygen is utilized as a cofactor for this reaction. Stabilized HIF1a transactivates a variety of hypoxia-responsive genes, therefore contributing to the cell adaptation to hypoxia ${ }^{52}$. Particularly, HIFla was found to be essential for hypoxia-mediated autophagy through promoting the expression of BNIP3 and BNIP3L, two BH3 domain-containing proteins, to release Beclin 1 from $\mathrm{Bcl}^{23}$. In the present data, however, HIFla shows minor effects on hypoxia-induced ULK1 R170me2s, suggesting that PRMT5 and KDM5C governs ULK1 R170me2s in an HIFlaindependent manner. Collectively, it is likely that ULK1 methylation and HIF1a hydroxylation synergistically regulates autophagy induction in response to hypoxic stress, by promoting the activation of ULK1 and Beclin 1-containing VPS34 complex and, respectively.

Autophagy, which enables the recycling of nutrients, amino acids and lipids, are often hijacked by tumor cells to counteract with stressful environment ${ }^{54}$. In this study, our findings illustrate a novel and important mechanism responsible for hypoxiainduced autophagy. Hypoxia-repressed KDM5C accumulates PRMT5-mediated ULK1 R170me2s, which results in ULK1 activation and autophagy induction. Inhibition of ULK1 R170me2s abolished hypoxia-triggered autophagy and impaired brain tumor development.

\footnotetext{
Methods

Materials. Rabbit polyclonal antibody recognizing phosphorylated ULK1 pT180 (1:1000), ULK1 R170me2s (1:1000) and ULK1 R170me2a (1: 1000) were customized from Boer Biotechnology (Chengdu, China). Peptides containing ULK1 pT180, ULK1 R170me2s, or ULK1 R170me2a were injected into rabbits. The rabbit serum was collected and purified using an affinity column conjugated with nonmodified peptide to exclude antibodies recognizing non-modified ULK1, followed by an affinity column conjugated with ULK1 pT180, ULK1 R170me2s or ULK1 R170me2a peptide to bind to and purify the antibodies. Antibodies were then eluted and concentrated.

Antibodies recognizing ULK1 (\#8054, 1:1000), HIF1a (\#36169, 1:1000), Tubulin (\#2125, 1:2000), LC3B (\#83506, 1:1000), p62 (\#88588, 1:1000), Beclin 1 (\#4122, 1:1000), Beclin 1 pS15 (\#84966, 1:500), Atg13 (\#13468, 1:1000), Atg13 pS355 (\#46329, 1:500), S6K pT421/pS424 (\#9204, 1:1000), Tomm20 (\#42406, 1:500), TSC1 (\#6935: 1:500) and AMPKa (\#5832, 1:1000) were obtained from Cell Signaling Technology. Antibodies recognizing ULK2 (ab97695, 1:1000), PRMT5 (ab109451, 1:1000), KDM5C (ab194288, 1:1000), BNIP3 (ab109362, 1:1000), S6K (ab32529, 1:1000), ACC (ab109368, 1:1000), ACC pS79 (ab68191, 1:500), ULK1 pS757 (ab229909, 1:500), and ULK1 pS555 (ab229537, 1:500) were obtained from Abcam. Antibody recognizing KDM4E (ABE1081, 1:1000) was obtained from Merck. Antibody against Flag (F2555, 1:5000), anti-Flag M2 agarose beads, streptavidin-conjugated agarose beads, a-ketoglutarate, dimethyl-aKG, DAPI, ATP, chloroquine and bovine serum albumin were purchased from Sigma. GFP-Trap beads were purchased from ChromoTek. IOX1 was purchased from Selleck Chemicals. HyFect transfection reagents were obtained from Denville Scientific. Recombinant Atg13 and Beclin 1 fusion proteins were purchased from Proteintech Group. $\left[\gamma^{-32} \mathrm{P}\right]-\mathrm{ATP}$ was purchased from MP Biomedicals.
}

DNA constructs and mutagenesis. PCR-amplified human ULK1, ULK2, KDM5C, PRMT5, Atg13, Beclin 1 and Atg14L were subcloned into pcDNA3.1/ hygro(+)-Flag, pcDNA3.1/hygro(+)-HA, pCDH-SFB, or pCold I (His) vector, respectively. Mutants were constructed using the QuikChange site-directed mutagenesis kit (Stratagene, La Jolla, CA). shRNA-resistant Beclin 1 contains mutation of c1332a, a1335c, a1338g and t1341c.

The following pGIPZ shRNAs were used: control shRNA, GCT TCT AAC ACC GGA GGT CTT; human ULK1 shRNA, TGC AAA GAA AAA CAC ACG T (targeting non-coding region); mouse ULK1 shRNA, AGT CTG CGT ACC ACC TGC T (not recognizing human ULK1); KDM5C shRNA \#1, TTC ATA GGG ATA AAC AAT G; KDM5C shRNA \#2, TTA TTA CAA CAT GAA CCC A; PRMT5 shRNA, AGT GTG TCA GCT ATT TCG G; HIFla shRNA, TAT AAA TAG ACT GCT TTA G; Beclin 1 shRNA, AAA ATT GTG AGG ACA CCC A. Atg13 shRNA was purchased from Sigma (Clone ID:NM_014741.3-2259s21c1, targeting non-coding region)

Cell culture and hypoxia treatment. LN229 GBM cells and 293T cells were obtained from ATCC. Huh7 HCC cells were obtained from JCRB Cell Bank. HOK cells were obtained from ScienCell. LN229, Huh7, and indicated MEF cells were maintained in Dulbecco's modified Eagle's medium supplemented with $10 \%$ fetal bovine serum. HOK cells were maintained in Oral Keratinocyte Medium (OKM, Cat. No. 2611). For generating shRNA-depleted stable cell lines, cells were transfected with shRNA plasmids and selected by puromycin. For generating geneexpressing stable cell lines, shRNA-depleted cells were infected with lentivirus carrying WT ULK1, ULK1 R170K or ULK1 T180A and selected by hygromycin. For hypoxia treatment, cells were incubated in a Ruskinn InvivO2 workstation under $1 \%$ oxygen concentration for indicated time, unless other oxygen concentrations were indicated.

Immunofluorescent staining. Cells were fixed and incubated with primary antibodies at a dilution of 1:100, fluorescence dye-conjugated secondary antibodies, and DAPI, according to standard protocols ${ }^{55}$. Cells were examined using a laser confocal microscope (Zeiss, Thornwood, NY). Cells were classified as having fragmented mitochondria when more than $50 \%$ of the total cellular organelles displayed a major axis shorter than $3 \mu \mathrm{m}^{56}$. The mitochondrial areas were quantified using Fiji software by calculating the Tomm20-positive areas of 200 cells.

Mass spectrometric analysis. Protein bands visualized via Coomassie Brilliant Blue staining was excised from SDS-PAGE gel and digested in $50 \mathrm{mM}$ ammonium bicarbonate buffer containing RapiGest (Waters Corporation) overnight at $37^{\circ} \mathrm{C}$ with $200 \mathrm{ng}$ of modified sequencing-grade trypsin (Promega). The digested samples were analyzed using high-sensitivity liquid chromatography tandem mass spectrometry with an Orbitrap Fusion Lumos mass spectrometer (Thermo Fisher Scientific).

BrdU incorporation assay. BrdU incorporation assay was performed using Cell Proliferation ELISA, BrdU (colorimetric) (Roche) following the manufacture's instruction. Briefly, cells were cultured in a 96-well microplate with $100 \mu \mathrm{l} /$ well medium. $10 \mu \mathrm{l} /$ well BrdU labeling solution was added in the culture and the cells were incubated for $8 \mathrm{~h}$ at $37^{\circ} \mathrm{C}$. After the labeling medium was removed, the cells were incubated with $200 \mu \mathrm{l} /$ well FixDenat at $25^{\circ} \mathrm{C}$ for $30 \mathrm{~min}, 100 \mu \mathrm{l} /$ well AntiBrdU-POD working solution at $25^{\circ} \mathrm{C}$ for $90 \mathrm{~min}$, and then $100 \mu \mathrm{l} /$ well Substrate Solution at $25^{\circ} \mathrm{C}$ for $20 \mathrm{~min}$. The absorbance of the samples was measured at $370 \mathrm{~nm}$.

Trypan blue exclusion assay. $0.1 \mathrm{ml}$ of the $0.4 \%$ trypan blue stock solution (Thermo Fisher Scientific) was added to $0.9 \mathrm{ml}$ cell suspension. 3 min later, $10 \mu \mathrm{l}$ cell suspension was loaded onto a hemacytometer, and the cells were examined immediately under a microscope at low magnification.

TUNEL assay. TUNEL assay was performed using TUNEL Assay Kit-HRP-DAB (Abcam) following the manufacture's instruction. Briefly, the specimen was sequentially incubated with Proteinase $\mathrm{K}$ at room temperature for $20 \mathrm{~min}$, 3\% hydrogen peroxide at room temperature for $5 \mathrm{~min}$, TdT Equilibration Buffer at room temperature for $30 \mathrm{~min}$, TdT Labeling Reaction Mix at $30^{\circ} \mathrm{C}$ for $90 \mathrm{~min}$, Stop Buffer at room temperature for $5 \mathrm{~min}$, Blocking Buffer at room temperature for $10 \mathrm{~min}$, and Conjugate solution at room temperature for $30 \mathrm{~min}$. The TUNEL signal was developed by incubation with $\mathrm{DAB}$ working solution at room temperature for $15 \mathrm{~min}$.

Genomic editing. Genomic mutations in LN229 cells were created using the CRISPR/Cas 9 system ${ }^{57}$. In a 24 -well plate, LN229 cells at $60 \%$ confluence were cotransfected with a donor template to introduce the mutations and a vector to express sgRNA and WT hSpCas9 tagged with green fluorescent protein. 48 hours after transfection, cells were trypsinized and diluted in a medium for single-cell seeding into a 96-well plate, and green fluorescent protein-positive cells were marked and subjected to genomic DNA extraction. 
Measurement of mitochondrial DNA content. Total DNA was extracted by using the QIAamp DNA mini kit (QIAGENE, Germantown, MD) according to the manufacturer's instructions. The levels of mitochondrial DNA D-Loop structure were measured by qRT-PCR, and normalized to genomic DNA-encoded $\beta$-actin. The sequences of the primers used were listed in Supplementary Table 1.

\section{Immunoprecipitation and immunoblot analysis. Extraction of proteins from} cultured cells was performed with a lysis buffer $(50 \mathrm{mM}$ Tris- $\mathrm{HCl}$ [pH 7.5], $0.1 \%$ SDS, $1 \%$ Triton X-100, $150 \mathrm{mM} \mathrm{NaCl}, 1 \mathrm{mM}$ dithiothreitol, $0.5 \mathrm{mM}$ EDTA, $100 \mu \mathrm{M}$ PMSF, $100 \mu \mathrm{M}$ leupeptin, $1 \mu \mathrm{M}$ aprotinin, $100 \mu \mathrm{M}$ sodium orthovanadate, $100 \mu \mathrm{M}$ sodium pyrophosphate, and $1 \mathrm{mM}$ sodium fluoride). Cell extracts were clarified by centrifugation at $13,400 \times g$, and the supernatants $(2 \mathrm{mg}$ protein/ml $)$ were subjected to immunoprecipitation with the indicated antibodies. After overnight incubation at $4{ }^{\circ} \mathrm{C}$, protein $\mathrm{A}$ or $\mathrm{G}$ agarose beads were added and left for an additional $3 \mathrm{~h}$. Immunocomplexes were washed with lysis buffer 3 times and then subjected to immunoblot analyses with corresponding antibodies ${ }^{58}$. The band intensity was quantified using the Image Lab software program (Bio-Rad).

Purification of recombinant proteins. Expression of PRMT5 proteins were induced in bacteria ${ }^{59}$. Briefly, BL21(DE3) cells expressing PRMT5 proteins were cultured in $250 \mathrm{ml}$ of $\mathrm{LB}$ medium and treated with isopropyl $\beta$-D-1 thiogalactopyranoside (IPTG) for $16 \mathrm{~h}$ at $30^{\circ} \mathrm{C}$ before lysis via sonication.

ULK1 and KDM5C proteins were expressed in 293 T cells. Briefly, a pcDNA3.1 plasmid $(10 \mu \mathrm{g})$ expressing recombinant proteins with tags were transfected into $293 \mathrm{~T}$ cells seeded in a $15 \mathrm{~cm}$-diameter dish with confluence of $70 \% .48 \mathrm{~h}$ after transfection, the cells were incubated with fresh DMEM supplemented with $10 \%$ bovine calf serum for $1 \mathrm{~h}$ and then harvested with a non-denaturing lysis buffer (20 mM Tris-HCl pH 8.0, $137 \mathrm{mM} \mathrm{NaCl}, 1 \% \mathrm{NP}-40$ ).

For purification of His-tagged proteins, cell lysates were loaded onto a Ni-NTA column (GE Healthcare Life Sciences) followed by washing with five column volumes of $20 \mathrm{mM}$ imidazole and subsequent elution with $250 \mathrm{mM}$ imidazole.

For purification of Flag-tagged proteins, cell lysates were loaded onto a column containing Anti-Flag M2 agarose affinity gel followed by washing with five column volumes of phosphate-buffered saline (PBS) and subsequent elution with $100 \mu \mathrm{g} / \mathrm{ml}$ Flag peptide. The proteins in $10-\mathrm{kDa}$ spin columns were desalted via washing twice with ice-cold PBS.

The proteins were then loaded onto a HiPrep 16/60 Sephacryl S-200 HR gel filtration column (GE Healthcare Life Sciences) to remove contaminated proteins. The purification efficiency was examined using SDS-PAGE and colloidal Coomassie Brilliant Blue (G-250) staining, with superior sensitivity (as low as $1 \mathrm{ng}$ of protein per band $)^{59}$.

TEM. Cells were fixed in $0.1 \%$ glutaraldehyde in $0.1 \mathrm{M}$ sodium cacodylate for $2 \mathrm{~h}$, postfixed with $1 \% \mathrm{OsO} 4$ for $1.5 \mathrm{~h}$, washed and finally stained for $1 \mathrm{~h}$ in $3 \%$ aqueous uranyl acetate. The samples were then rinsed with water again, dehydrated with graded alcohol (50\%, 75\% and 95-100\% alcohol) and embedded in Epon-Araldite resin (Canemco, 034). Ultrathin sections were cut on a Reichert Ultramicrotome, counterstained with $0.3 \%$ lead citrate and examined on a Philips EM420 transmission electron microscope. The cells with autophagic vacuoles were defined as cells that had 5 or more autophagic vacuoles ${ }^{60}$. Values for the area occupied by autophagic vacuoles and the cytoplasm were obtained with Image Pro Plus version 3 .

In vitro methylation assay. In vitro methylation assay was performed according to the previous report ${ }^{61}$. Briefly, $500 \mathrm{ng}$ purified PRMT5 protein and $200 \mathrm{ng}$ purified ULK1 protein were mixed with $50 \mu$ methylation buffer $(20 \mathrm{mM}$ Tris [pH 8.8 ], $4 \mathrm{mM}$ EDTA, $1 \mathrm{mM}$ PMSF, $0.5 \mathrm{mM}$ DTT and $5 \mathrm{mM} \mathrm{SAM}$ ), and incubated at $30^{\circ} \mathrm{C}$ for $1 \mathrm{~h}$. The reaction was stopped by adding SDS loading buffer, and the proteins were resolved on SDS-PAGE gels.

In vitro demethylation assay. $500 \mathrm{ng}$ purified $\mathrm{KDM} 5 \mathrm{C}$ protein and $200 \mathrm{ng}$ purified ULK1 protein were incubated in $100 \mu \mathrm{l}$ of demethylase reaction buffer containing $20 \mathrm{mM}$ Tris- $\mathrm{HCl}(\mathrm{pH} 7.5), 150 \mathrm{mM} \mathrm{KCl}, 50 \mathrm{mM} \mathrm{Fe}\left(\mathrm{NH}_{4}\right)_{2}\left(\mathrm{SO}_{4}\right)_{2}, 1 \mathrm{mM}$ aketoglutarate, and $1 \mathrm{mM}$ ascorbate for $1 \mathrm{~h}$ at $37^{\circ} \mathrm{C}^{62}$. Reactions were terminated by boiling for $5 \mathrm{~min}$ in SDS sample loading buffer.

ULK1 kinase assay. In vitro ULK1 kinase assay was performed following previous report ${ }^{15}$. For ULK1-mediated phosphorylation of Atg13 or Beclin 1, 20 ng modified or non-modified ULK1 proteins were mixed with $10 \mathrm{ng}$ Atg13 or $10 \mathrm{ng}$ Beclin 1 proteins were incubated in $25 \mu$ of kinase buffer $(50 \mathrm{mM}$ Tris- $\mathrm{HCl}$ [pH 7.5], $100 \mathrm{mM} \mathrm{KCl}, 50 \mathrm{mM} \mathrm{MgCl}_{2}, 50 \mathrm{mM} \mathrm{MnCl}, 1 \mathrm{mM} \mathrm{Na}_{3} \mathrm{VO}_{4}, 1 \mathrm{mM}$ DTT, $5 \%$ glycerol, $0.5 \mathrm{mM} \mathrm{ATP}$ ) at $37^{\circ} \mathrm{C}$ for $1 \mathrm{~h}$. For ULK1 autophosphorylation, $5 \mathrm{ng}$ modified or non-modified ULK1 proteins were incubated in $25 \mu \mathrm{l}$ of kinase buffer at $37^{\circ} \mathrm{C}$ for $10 \mathrm{~min}$. ULK1 activity assay was performed using ADP-Glo ${ }^{\mathrm{mc}}$ Kinase Assay + ULK1 Kinase Enzyme System (Promega, Madison, WI, USA) ${ }^{63}$.

VPS34 lipid kinase assay. Atg14L-associated VPS34 complex was immunoprecipitated, washed with PBS, followed by three washes with the reaction buffer. The protein complex was incubated in $60 \mu \mathrm{L}$ of reaction buffer $(10 \mathrm{mM}$ Tris- $\mathrm{HCl}[\mathrm{pH}$ 7.4], $100 \mathrm{mM} \mathrm{NaCl}, 1 \mathrm{mM}$ EDTA) supplemented with $10 \mathrm{mM} \mathrm{MnCl} 2,20 \mu \mathrm{g}$ phosphatidylinositol, $0.5 \mathrm{mM} \mathrm{ATP}$, and $10 \mu \mathrm{Ci}\left[\gamma^{3}{ }^{32} \mathrm{P}\right] \mathrm{ATP}$ at ambient temperature for $30 \mathrm{~min}$. The reaction was quenched by adding $20 \mu \mathrm{L}$ of $8 \mathrm{M} \mathrm{HCl}$ and extracted with $160 \mu \mathrm{L}$ chloroform/methanol (1:1). Extracted phospholipid products were separated on TLC using a coated silica gel and a solvent composed of chloroform/ methanol $/ \mathrm{H}_{2} \mathrm{O} / \mathrm{NH}_{4} \mathrm{OH}(\mathrm{v} / \mathrm{v} / \mathrm{v} / \mathrm{v}, 9: 7: 1.7: 0.3)$. The TLC plates were dried and exposed by autoradiography to visualize $\mathrm{PI}(3) \mathrm{P}$ production.

Measurement of cellular OCR. Briefly, $3 \times 10^{4}$ cells were plated onto XF24 plates in DMEM (0.5\% BCS, $10 \mathrm{mM}$ glucose, $2 \mathrm{mM}$ glutamine) (Seahorse Bioscience, North Billerica, MA) and incubated at in $21 \%$ or $1 \%$ oxygen tension for indicated time. The medium was then replaced with $675 \mu \mathrm{l}$ of unbuffered assay medium (Seahorse Bioscience) supplemented with $2 \mathrm{mM}$ glutamine, $10 \mathrm{mM}$ glucose $(\mathrm{pH}$ was adjusted to 7.4 using $0.5 \mathrm{mM}$ sodium hydroxide). Cellular OCR were recorded using the XF24 plate reader. Homogeneous plating and cell counts were assessed by fixing the cells with $10 \%$ trichloroacetic acid for $1 \mathrm{~h}$ at $4{ }^{\circ} \mathrm{C}$ and then staining the fixed cells with a $0.47 \%$ solution of sulforhodamine $\mathrm{B}^{64}$.

Mouse xenograft model. Four-week-old female athymic nude $\mathrm{BALB} / \mathrm{c}$ mice were used in this study. Mice were housed under ambient temperature of $24 \pm 2{ }^{\circ} \mathrm{C}$, circulating air, constant humidity of $50 \pm 10 \%$ and a $12 \mathrm{~h}: 12 \mathrm{~h}$ light/dark cycle. $1 \times 10^{5}$ cells with knockin mutations were suspended in $5 \mu \mathrm{l}$ of Dulbecco's modified Eagle's medium into mice $(n=8)$. For hypoxia treatment, mice were raised in a controlled oxygen tension (11\%) chamber. Animals were sacrificed 28 days after injection. The brain of each mouse was dissected, which is followed by fixation in $4 \%$ formaldehyde and embedment in paraffin. The tumor volume was calculated using the formula: $V=1 / 2 a^{2} b$ ( $V$ : volume, $a$ : shortest diameter, $b$ : longest diameter) The animals were treated in accordance with relevant institutional and national guidelines and regulations. The use of animals was approved by the institutional review board of Chengdu Medical College.

Zebrafish xenograft model. Zebrafish xenograft was established using embryos of $\mathrm{Tg}$ (flk1: EGFP) zebrafish strain obtained 48 hours postfertilization, following previous report ${ }^{65}$. Zebrafish were maintained under temperature at $28^{\circ} \mathrm{C}, \mathrm{pH}$ 7.2-7.4, and a $14 \mathrm{~h}$-on/10 h-off light cycle. Cells were labeled with $2 \mu \mathrm{M}$ CellTracker $^{\text {min }}$ CM-Dil Dye (Thermo Fisher Scientific), washed with PBS, and loaded into a borosilicate glass needle pulled by a Flaming/Brown micropipette puller (Narishige, Japan, PN-30). Zebrafish embryos, $48 \mathrm{~h}$ postfertilization, were anesthetized by $0.003 \%$ tricaine. $20 \mathrm{~nL}$ suspension containing about 15 cells were implanted into the pericardial cavity of zebrafish embryo by using an electronically regulated air-pressure microinjector (Harvard Apparatus, NY, PL1-90). After injection, digital micrographs were taken with a Zeiss Imager. Z1 fluorescence microscope (Carl Zeiss Microimaging Inc., Germany) equipped with an AxioCam MRc5 digital CCD camera (Carl Zeiss Microimaging Inc., Germany). The number of CM-Dil-labeled cells was evaluated by automated cell counting via custom 66,67 . Those zebrafish embryos with 15 cells were selected for each group. Zebrafish were maintained in a 1-liter standard plastic aquarium with a lid. For hypoxia treatment, nitrogen gas tank was switched on and the air saturation level shown on the oxygen regulator was set to $7.5 \%{ }^{45}$. 5 days after infection, zebrafish without cell dissemination to the posterior part were selected ( $n=10$ for each group), and the CM-Dil-labeled cells were quantified.

Histologic evaluation and immunohistochemical staining. The immunohistochemical staining was performed using the VECTASTAIN ABC kit (VECTOR LABORATORIES, CA) according to manufacturer's instructions. We quantitatively scored the tissue sections according to the percentage of positive cells and staining intensity, as previously defined ${ }^{68}$. We assigned the following proportion scores: 0 if $0 \%$ of the tumor cells showed positive staining, 1 if $0 \%$ to $10 \%, 2$ if $11 \%$ to $30 \%, 3$ if $31 \%$ to $70 \%$, and 4 if $71 \%$ to $100 \%$. We also rated the intensity of staining on a scale of 0 to $4: 0$, negative; 1 , weak; 2 , moderate; 3 , strong; 4 , very strong. We then multiply the proportion and intensity scores to obtain a total score (range: 0-16). The use of human GBM tumor specimens was approved by the Institutional Review Board at Chengdu Medical college.

Statistics and reproducibility. All experiments were repeated independently with similar results for three times, and all data represent the mean \pm standard deviation (SD) of three independent experiments, unless otherwise specified. Differences in means were considered statistically significant at $P<0.05$. Bonferroni correction was used for multiple hypothesis correction (requiring $p<0.05 / \mathrm{N}$, with $\mathrm{N}$ indicating the number of comparisons) to prevent the reporting of false positive results. GraphPad Prism 7 software was used for statistical analyses. 
Reporting summary. Further information on research design is available in the Nature Research Reporting Summary linked to this article.

\section{Data availability}

All the data supporting the findings of this study are available within the article and its Supplementary information files. The proteomic data generated in this study have been deposited in the ProteomeXchange Consortium via the iProX proteomic data repository under the accession number PXD031209. All relevant data are available upon reasonable request. Source data are provided with this paper.

Received: 27 March 2020; Accepted: 10 February 2022;

Published online: 04 March 2022

\section{References}

1. Hermes-Lima, M. et al. Preparation for oxidative stress under hypoxia and metabolic depression: revisiting the proposal two decades later. Free Radic. Biol. Med. 89, 1122-1143 (2015).

2. Semenza, G. L. Hypoxia-inducible factors: coupling glucose metabolism and redox regulation with induction of the breast cancer stem cell phenotype. EMBO J. 36, 252-259 (2017).

3. Mansfield, K. D. et al. Mitochondrial dysfunction resulting from loss of cytochrome c impairs cellular oxygen sensing and hypoxic HIF-alpha activation. Cell Metab. 1, 393-399 (2005)

4. Vaupel, P., Kallinowski, F. \& Okunieff, P. Blood flow, oxygen and nutrient supply, and metabolic microenvironment of human tumors: a review. Cancer Res. 49, 6449-6465 (1989).

5. Rabinowitz, J. D. \& White, E. Autophagy and metabolism. Science 330, 1344-1348 (2010).

6. Parzych, K. R. \& Klionsky, D. J. An overview of autophagy: morphology, mechanism, and regulation. Antioxid. Redox Signal 20, 460-473 (2014).

7. Ravanan, P., Srikumar, I. F. \& Talwar, P. Autophagy: the spotlight for cellular stress responses. Life Sci. 188, 53-67 (2017).

8. Yu, S. et al. Autophagy in the "inflammation-carcinogenesis" pathway of liver and HCC immunotherapy. Cancer Lett. 411, 82-89 (2017).

9. Yang, A. et al. Autophagy sustains pancreatic cancer growth through both cellautonomous and nonautonomous mechanisms. Cancer Discov. 8, 276-287 (2018).

10. Lin, Y. T. et al. Capsaicin induces autophagy and apoptosis in human nasopharyngeal carcinoma cells by downregulating the PI3K/AKT/mTOR Pathway. Int. J. Mol. Sci. 18, https://doi.org/10.3390/ijms18071343 (2017).

11. Sannigrahi, M. K., Singh, V., Sharma, R., Panda, N. K. \& Khullar, M. Role of autophagy in head and neck cancer and therapeutic resistance. Oral. Dis. 21, 283-291 (2015).

12. Mizushima, N. The role of the Atg1/ULK1 complex in autophagy regulation. Curr. Opin. cell Biol. 22, 132-139 (2010).

13. Zachari, M. \& Ganley, I. G. The mammalian ULK1 complex and autophagy initiation. Essays Biochem. 61, 585-596 (2017).

14. Ganley, I. G. et al. ULK1.ATG13.FIP200 complex mediates mTOR signaling and is essential for autophagy. J. Biol. Chem. 284, 12297-12305 (2009).

15. Russell, R. C. et al. ULK1 induces autophagy by phosphorylating Beclin-1 and activating VPS34 lipid kinase. Nat. cell Biol. 15, 741-750 (2013).

16. Funderburk, S. F., Wang, Q. J. \& Yue, Z. The Beclin 1-VPS34 complex-at the crossroads of autophagy and beyond. Trends cell Biol. 20, 355-362 (2010).

17. Kim, J. et al. Differential regulation of distinct Vps34 complexes by AMPK in nutrient stress and autophagy. Cell 152, 290-303 (2013).

18. Volinia, S. et al. A human phosphatidylinositol 3-kinase complex related to the yeast Vps34p-Vps15p protein sorting system. EMBO J. 14, 3339-3348 (1995).

19. Tanida, I., Minematsu-Ikeguchi, N., Ueno, T. \& Kominami, E. Lysosomal turnover, but not a cellular level, of endogenous LC3 is a marker for autophagy. Autophagy 1, 84-91 (2005).

20. Pankiv, S. et al. p62/SQSTM1 binds directly to Atg8/LC3 to facilitate degradation of ubiquitinated protein aggregates by autophagy. J. Biol. Chem. 282, 24131-24145 (2007).

21. Jiang, P. \& Mizushima, N. LC3- and p62-based biochemical methods for the analysis of autophagy progression in mammalian cells. Methods 75, 13-18 (2015).

22. Kim, J., Kundu, M., Viollet, B. \& Guan, K. L. AMPK and mTOR regulate autophagy through direct phosphorylation of Ulk1. Nat. cell Biol. 13, 132-141 (2011).

23. Jung, C. H., Ro, S. H., Cao, J., Otto, N. M. \& Kim, D. H. mTOR regulation of autophagy. FEBS Lett. 584, 1287-1295 (2010).

24. DeYoung, M. P., Horak, P., Sofer, A., Sgroi, D. \& Ellisen, L. W. Hypoxia regulates TSC1/2-mTOR signaling and tumor suppression through REDD1mediated 14-3-3 shuttling. Genes Dev. 22, 239-251 (2008).
25. Mungai, P. T. et al. Hypoxia triggers AMPK activation through reactive oxygen species-mediated activation of calcium release-activated calcium channels. Mol. Cell. Biol. 31, 3531-3545 (2011).

26. Kundu, M. et al. Ulk1 plays a critical role in the autophagic clearance of mitochondria and ribosomes during reticulocyte maturation. Blood 112, 1493-1502 (2008)

27. Feng, J. et al. PTEN arginine methylation by PRMT6 suppresses PI3K-AKT signaling and modulates pre-mRNA splicing. Proc. Natl. Acad. Sci. USA 116, 6868-6877 (2019)

28. Larsen, S. C. et al. Proteome-wide analysis of arginine monomethylation reveals widespread occurrence in human cells. Sci. Signal. 9, rs9 (2016).

29. Bedford, M. T. \& Richard, S. Arginine methylation an emerging regulator of protein function. Mol. Cell 18, 263-272 (2005).

30. Marjon, K. et al. MTAP Deletions in cancer create vulnerability to targeting of the MAT2A/PRMT5/RIOK1 axis. Cell Rep. 15, 574-587 (2016).

31. Maes, T., Carceller, E., Salas, J., Ortega, A. \& Buesa, C. Advances in the development of histone lysine demethylase inhibitors. Curr. Opin. Pharmacol. 23, 52-60 (2015).

32. Walport, L. J. et al. Arginine demethylation is catalysed by a subset of JmjC histone lysine demethylases. Nat. Commun. 7, 11974 (2016).

33. Iwase, $\mathrm{S}$. et al. The X-linked mental retardation gene SMCX/JARID1C defines a family of histone H3 lysine 4 demethylases. Cell 128, 1077-1088 (2007).

34. Klose, R. J., Kallin, E. M. \& Zhang, Y. JmjC-domain-containing proteins and histone demethylation. Nat. Rev. Genet. 7, 715-727 (2006).

35. Nazio, F. et al. mTOR inhibits autophagy by controlling ULK1 ubiquitylation, self-association and function through AMBRA1 and TRAF6. Nat. Cell Biol. 15, 406-416 (2013).

36. Joo, J. H. et al. Hsp90-Cdc37 chaperone complex regulates Ulk1- and Atg13mediated mitophagy. Mol. Cell 43, 572-585 (2011).

37. Russell, R. C. et al. ULK1 induces autophagy by phosphorylating Beclin-1 and activating VPS34 lipid kinase. Nat. cell Biol. 15, 741-750 (2013).

38. Yeh, Y. Y., Wrasman, K. \& Herman, P. K. Autophosphorylation within the Atg1 activation loop is required for both kinase activity and the induction of autophagy in Saccharomyces cerevisiae. Genetics 185, 871-882 (2010).

39. Liu, L. et al. Mitochondrial outer-membrane protein FUNDC1 mediates hypoxiainduced mitophagy in mammalian cells. Nat. cell Biol. 14, 177-185 (2012).

40. Chai, P. et al. USP19 promotes hypoxia-induced mitochondrial division via FUNDC1 at ER-mitochondria contact sites. J. Cell Biol. 220, https://doi.org/ 10.1083/jcb.202010006 (2021).

41. Kanaji, S., Iwahashi, J., Kida, Y., Sakaguchi, M. \& Mihara, K. Characterization of the signal that directs Tom 20 to the mitochondrial outer membrane. J. Cell Biol. 151, 277-288 (2000).

42. Tormos, K. V. \& Chandel, N. S. Inter-connection between mitochondria and HIFs. J. Cell. Mol. Med. 14, 795-804 (2010).

43. Giatromanolaki, A. et al. BNIP3 expression is linked with hypoxia-regulated protein expression and with poor prognosis in non-small cell lung cancer. Clin. Cancer Res. 10, 5566-5571 (2004).

44. Okami, J., Simeone, D. M. \& Logsdon, C. D. Silencing of the hypoxiainducible cell death protein BNIP3 in pancreatic cancer. Cancer Res. 64 5338-5346 (2004)

45. Rouhi, P. et al. Hypoxia-induced metastasis model in embryonic zebrafish. Nat. Protoc. 5, 1911-1918 (2010).

46. Bristow, R. G. \& Hill, R. P. Hypoxia and metabolism. Hypoxia, DNA repair and genetic instability. Nat. Rev. Cancer 8, 180-192 (2008).

47. Wysocka, J., Allis, C. D. \& Coonrod, S. Histone arginine methylation and its dynamic regulation. Front. Biosci.11, 344-355 (2006).

48. Ng, S. S., Yue, W. W., Oppermann, U. \& Klose, R. J. Dynamic protein methylation in chromatin biology. Cell. Mol. Life Sci. 66, 407-422 (2009).

49. Lee, D. Y., Teyssier, C., Strahl, B. D. \& Stallcup, M. R. Role of protein methylation in regulation of transcription. Endocr. Rev. 26, 147-170 (2005)

50. Ancelin, K. et al. Blimp1 associates with Prmt5 and directs histone arginine methylation in mouse germ cells. Nat. cell Biol. 8, 623-630 (2006).

51. Rabanal-Ruiz, Y., Otten, E. G. \& Korolchuk, V. I. mTORC1 as the main gateway to autophagy. Essays Biochem. 61, 565-584 (2017).

52. Keith, B., Johnson, R. S. \& Simon, M. C. HIF1alpha and HIF2alpha: sibling rivalry in hypoxic tumour growth and progression. Nat. Rev. Cancer 12, 9-22 (2011)

53. Bellot, G. et al. Hypoxia-induced autophagy is mediated through hypoxiainducible factor induction of BNIP3 and BNIP3L via their BH3 domains. Mol. Cell. Biol. 29, 2570-2581 (2009).

54. Parks, S. K., Cormerais, Y. \& Pouyssegur, J. Hypoxia and cellular metabolism in tumour pathophysiology. J. Physiol. 595, 2439-2450 (2017).

55. Fang, D. et al. Phosphorylation of beta-catenin by AKT promotes beta-catenin transcriptional activity. J. Biol. Chem. 282, 11221-11229 (2007).

56. Costa, V. et al. Mitochondrial fission and cristae disruption increase the response of cell models of Huntington's disease to apoptotic stimuli. EMBO Mol. Med. 2, 490-503 (2010).

57. Sander, J. D. \& Joung, J. K. CRISPR-Cas systems for editing, regulating and targeting genomes. Nat. Biotechnol. 32, 347-355 (2014). 
58. Lu, Z. et al. Activation of protein kinase $\mathrm{C}$ triggers its ubiquitination and degradation. Mol. Cell. Biol. 18, 839-845 (1998).

59. Xia, Y. et al. c-Jun downregulation by HDAC3-dependent transcriptional repression promotes osmotic stress-induced cell apoptosis. Mol. Cell 25, 219-232 (2007).

60. Liu, R. et al. Itraconazole suppresses the growth of glioblastoma through induction of autophagy: involvement of abnormal cholesterol trafficking. Autophagy 10, 1241-1255 (2014).

61. Amente, S. et al. Identification of proteins interacting with the RNAPII FCP1 phosphatase: FCP1 forms a complex with arginine methyltransferase PRMT5 and it is a substrate for PRMT5-mediated methylation. FEBS Lett. 579, 683-689 (2005).

62. Whetstine, J. R. et al. Reversal of histone lysine trimethylation by the JMJD2 family of histone demethylases. Cell 125, 467-481 (2006).

63. Balzano, D., Santaguida, S., Musacchio, A. \& Villa, F. A general framework for inhibitor resistance in protein kinases. Chem. Biol. 18, 966-975 (2011).

64. Li, X. et al. Mitochondria-translocated PGK1 functions as a protein kinase to coordinate glycolysis and the TCA cycle in tumorigenesis. Mol. Cell 61, 705-719 (2016).

65. Zhao, C. et al. Distinct contributions of angiogenesis and vascular co-option during the initiation of primary microtumors and micrometastases. Carcinogenesis 32, 1143-1150 (2011).

66. Teng, Y. et al. Evaluating human cancer cell metastasis in zebrafish. BMC Cancer 13, 453 (2013).

67. Schindelin, J. et al. Fiji: an open-source platform for biological-image analysis. Nat. methods 9, 676-682 (2012).

68. Lee, J. H. et al. EGFR-Phosphorylated platelet isoform of phosphofructokinase 1 promotes PI3K activation. Mol. Cell 70, 197-210.e197 (2018).

\section{Acknowledgements}

We thank Yi Zhong (Proteomics-Metabolomics Platform, Core Facilities of West China Hospital) for proteomic analyses. This work was supported by National Natural Science Foundation of China grants 81872218, 81772692, 81773143, and 82001047; Scientific Research Program for Young Talents of China National Nuclear Corporation 2020CNNC74; Sichuan Science \& Technology Program 2020YFSY0009, 2018JY0368, and 2019YJ0371; Sichuan Province Science and technology support program 2019YFS0110; Research Funding from West China School/Hospital of Stomatology Sichuan University RCDWJS2020-8; Fok Ying Tong Education Foundation grant 161036; and the National Research Foundation of Korea grant funded by the Korea government (MSIT) 2020R1C1C1011350.

\section{Author contributions}

R.L. conceived and designed the study; J.L., T.Z., T.R., X.L., Y.H., J.S.L., J-H.L., J.S. and M.L. performed the experiments; and R.L. wrote the paper, with comments from all authors.

\section{Competing interests}

The authors declare no competing interests.

\section{Additional information}

Supplementary information The online version contains supplementary material available at https://doi.org/10.1038/s41467-022-28831-6.

Correspondence and requests for materials should be addressed to Rui Liu.

Peer review information Nature Communications thanks the anonymous reviewer(s) for their contribution to the peer review of this work.

Reprints and permission information is available at http://www.nature.com/reprints

Publisher's note Springer Nature remains neutral with regard to jurisdictional claims in published maps and institutional affiliations.

cc (i) Open Access This article is licensed under a Creative Commons Attribution 4.0 International License, which permits use, sharing, adaptation, distribution and reproduction in any medium or format, as long as you give appropriate credit to the original author(s) and the source, provide a link to the Creative Commons license, and indicate if changes were made. The images or other third party material in this article are included in the article's Creative Commons license, unless indicated otherwise in a credit line to the material. If material is not included in the article's Creative Commons license and your intended use is not permitted by statutory regulation or exceeds the permitted use, you will need to obtain permission directly from the copyright holder. To view a copy of this license, visit http://creativecommons.org/ licenses/by/4.0/.

(c) The Author(s) 2022 